\title{
A Finite-Volume Method for Fluctuating Dynamical Density Functional Theory
}

\author{
Antonio Russo ${ }^{\mathrm{a}, *}$, Sergio P. Perez ${ }^{\mathrm{a}, \mathrm{b}, *}$, Miguel A. Durán-Olivencia ${ }^{\mathrm{a}}$, Peter Yatsyshin ${ }^{\mathrm{a}}$, José A. Carrillo ${ }^{\mathrm{b}}$, Serafim \\ Kalliadasis $^{\mathrm{a}}$ \\ ${ }^{a}$ Department of Chemical Engineering, Imperial College London, London, United Kingdom \\ ${ }^{b}$ Department of Mathematics, Imperial College London, London, United Kingdom
}

\section{A R T I C LE INFO}

\section{Article history:}

Received 1 Sep 2019

Received in final form 2019

Accepted 2019

Available online 2019

Communicated by Editor name

Stochastic partial differential equation, finite-volume, fluctuating dynamical density functional theory

\begin{abstract}
A B S T R A C T
In this work we introduce a finite-volume numerical scheme for solving stochastic gradient flow equations. Such equations are of crucial importance within the framework of fluctuating hydrodynamics and dynamic density functional theory. Our proposed scheme deals with general free-energy functionals, including, for instance, external fields or interaction potentials. This allows us to simulate a range of physical phenomena where thermal fluctuations play a crucial role, such as nucleation and further energy-barrier crossing transitions. A positivity-preserving algorithm for the density is derived based on a hybrid space discretization of the deterministic and the stochastic terms and different implicit and explicit time integrators. We show through numerous applications that not only our scheme is able to accurately reproduce the statistical properties (structure factor and correlations) of the physical system, but, because of the multiplicative noise, it allows us to simulate energy barrier crossing dynamics, which cannot be captured by mean field approaches.
\end{abstract}

(C) 2020 Elsevier Inc. All rights reserved.

\section{Introduction}

The dynamics of fluids has shown to be a complex subject of study, among other things due to the variety of time scales involved. This is why the time evolution of such fluids is commonly studied via numerical simulations, either at molecular scale, by using molecular dynamics (MD) or Monte Carlo (MC) simulation; or at macro scale, by utilising deterministic models based on the conservation of fundamental quantities, namely mass, momentum and energy. While atomistic simulations take into account thermal fluctuations, they come with an important drawback, the enormous computational cost of having to resolve at least three degrees of freedom per particle. Despite drastic improvements in computational power over the last few decades, atomistic simulations are only applicable for small fluid volumes. There are also other challenges with such techniques, e.g. the use of a proper thermostat when running non-equilibrium simulations at constant temperature [1]. On the contrary, the convenience of partial differential

\footnotetext{
${ }^{*}$ A. Russo and S. P. Perez are co-first and contributed equally to this work. S. P. Perez is the corresponding author:

e-mail: sergio.perez15@imperial.ac.uk (Sergio P. Perez)
} 
equations (PDEs), such as continuity and Navier-Stokes, is enormous as they are amenable to both analytical and numerical scrutiny, with numerical simulations being less computationally expensive than MD-MC. However, continuous models based upon PDEs cannot account for the stochastic nature observed in real systems. Fortunately, there is still an approach which lives at the crossroad of mesoscale, namely fluctuating hydrodynamics (FH). Firstly proposed by Landau and Lifshitz [2], FH is formulated in terms of stochastic PDEs which aim at extending Navier-Stokes equations to include thermal fluctuations. FH can then be used to simulate systems undergoing energy-barrier crossing transitions, such as nucleation, which are impossible to describe within the mean field approximation.

The first phenomenological description of FH is due to Landau and Lifshitz [2], who included additive stochastic flux terms in the Navier-Stokes equations - we shall refer to these equations as the Landau-Lifshitz-Navier-Stokes (LLNS) equations. A remarkable effort has been made ever since trying to connect FH with MD from first principles $[3,4,5,6,7,8,9]$. Some of the most widely known attempts to formalise such a connection are the works of Dean [7] and Kawasaki [6]. Theirs provide a formal derivation of the stochastic time-evolution equation for the "density" field of a system of Brownian particles. Nevertheless, their derivation ends up with a time-evolution equation for the microscopic density field, which is nothing but a re-writing of the Brownian equations by using Itô's lemma. For this reason, the Dean-Kawasaki equation has been actively criticised and just seen as a toy model not representing a proper proof of the overdamped FH equation. However, this model cannot be employed to describe macroscopic quantities, such as density and momentum fields which are obtained by ensemble averaging the corresponding microscopic quantities [10], and thus remains disconnected from the original Landau-Lifshitz theory. And it is this disconnection that has led to the misconception that the so-called "Dean-Kawasaki" model describes the evolution of macroscopic observables.

In a recent work [11], a bottom-up derivation of the fluctuating hydrodynamics for a system of Brownian particles has been posed. It provided a new formulation from first principle of the governing equations for macroscopic observables in the framework of classical dynamic density functional theory (DDFT). It is worth mentioning that the field of DDFT has gained a lot of traction since the first phenomenological derivations proposed in [12, 13]. Several rigorous derivations have been put forward including effects such as inertia, hydrodynamic interactions and orientation of particles. These derivations have been proposed both for the overdamped and inertial regimes, and we refer the reader to $[14,15,16,17,18]$ for more details about those derivations.

The formulation proposed in [11] allows for a rigorous and systematic derivation of FH but also fluctuating DDFT (FDDFT) which includes the effects of thermal fluctuations on the mean-field DDFT. In that work, it is also shown how the classical DDFT is the most-likely realisation of FDDFT, thus providing closure to a long standing debate in the classical DFT community about the inclusion of fluctuations in DFT. Also, the derivation by DuránOlivencia et al. [11] stays in tune with the original intuitive treatment of Landau and Lifshitz and at the same time alleviates the misconceptions with the Dean-Kawasaki model. As a remark, it should be noticed that LLNS equations describe a full system of particles, while FDDFT governs the time-evolution of density and momentum fields of subcomponents of a system, e.g. of colloidal particles in a bath. Because of the momentum exchange between colloidal and bath particles, the total momentum in FDDFT for colloidal particles is not conserved, being affected by thermal fluctuations and friction exerted by the bath. Let us note here that both classical DFT and DDFT, embedded with either exact or approximated models for the density-dependent Helmholtz free energy functional [19], has already shown its enhanced capabilities in the study of complex system at the nano- and microscale [20, 14, 21]. Recent advances in classical DFT have extended its applicability to a wide spectrum of applications from nucleation of colloids and macro-molecules [22, 23, 24] to fluids in confined geometries [25, 26, 27] and wetting phenomena $[28,29,30]$. But also highly non-uniform systems such as dense liquid droplets and solid clusters [31].

The FDDFT framework in Ref. [11] derived for the general case of arbitrarily shaped and thermalized particles consists of two stochastic PDEs for the number density $\rho$ (known also as particles state probability function) and velocity $\mathbf{v}$ fields:

$$
\begin{aligned}
& \partial_{t} \rho(\mathbf{r}, t)+\nabla_{\mathbf{r}} \cdot(\rho(\mathbf{r}, t) v(\mathbf{r}, t))=0, \\
& \partial_{t}(m \rho(\mathbf{r}, t) \mathbf{v}(\mathbf{r}, t))+\nabla_{\mathbf{r}} \cdot(m \rho(\mathbf{r}, t) \mathbf{v}(\mathbf{r}, t) \otimes \mathbf{v}(\mathbf{r}, t))+\rho(\mathbf{r}, t) \nabla_{\mathbf{r}} \frac{\delta \mathcal{E}[\rho]}{\delta \rho(\mathbf{r}, t)}+m \gamma \rho(\mathbf{r}, t) \mathbf{v}(\mathbf{r}, t)+\sqrt{k_{B} T m \gamma \rho(\mathbf{r}, t)} \boldsymbol{W}(\mathbf{r}, t)=0,
\end{aligned}
$$

where $m$ is the mass of the particles, $\mathcal{E}[\rho]$ is the density-dependent free-energy functional, $\gamma$ is a friction parameter describing the interactions between the particles and the bath, $k_{B}$ is the Boltzmann constant, $T$ is the temperature and 
$\boldsymbol{W}$ is a vector of Gaussian stochastic processes delta-correlated in space and time, i.e.

$$
\begin{aligned}
\langle\boldsymbol{W}(\mathbf{r}, t)\rangle & =0, \\
\left\langle\boldsymbol{W}(\mathbf{r}, t), \boldsymbol{W}\left(\mathbf{r}^{\prime}, t^{\prime}\right)\right\rangle & =2 \delta\left(t-t^{\prime}\right) \delta\left(\mathbf{r}-\mathbf{r}^{\prime}\right) .
\end{aligned}
$$

In the strong damping limit $\left(m^{-1} \gamma \rightarrow \infty\right)$, the high friction between the particles and the bath causes the characteristic time scale of the momentum dynamics to be much shorter than the density one [15,11]. Thus, as a first approximation, the contributions of the terms $\nabla_{\mathbf{r}} \cdot(m \rho \mathbf{v} \otimes \mathbf{v})$ and $\partial_{t}(m \rho \mathbf{v})$ can be neglected. As a result, one obtains the stochastic time-evolution equation for the density field, referred to as overdamped FDDFT [11, 32]:

$$
\partial_{t} \rho(\mathbf{r}, t)=\nabla_{\mathbf{r}} \cdot\left((m \gamma)^{-1} \rho(\mathbf{r}, t) \nabla_{\mathbf{r}} \frac{\delta \mathcal{E}[\rho]}{\delta \rho(\mathbf{r}, t)}\right)+\nabla_{\mathbf{r}} \cdot\left(\sqrt{k_{B} T(m \gamma)^{-1} \rho(\mathbf{r}, t)} \boldsymbol{W}(\mathbf{r}, t)\right) .
$$

Equation (5) may be seen as a stochastic version of the gradient flow equation previously studied, for instance, in Refs [33, 34]. As we later discuss in further detail, Eq. (5) reduces to the stochastic diffusion equation [35] when considering a system of non-interacting particles (ideal gas), whose free energy would be $\mathcal{E}[\rho]=\int \rho(\log \rho-1) d \mathbf{r}$. However, the presence of a more general functional $\mathcal{E}[\rho]$ allows in principle to introduce non-linear diffusion, external force fields and interparticle interactions. It is also worth mentioning that Eq. (5) is not well-posed due to the high irregularity originated in the stochastic fluxes and the multiplicity of the noise. This difficulty is typically overcome by introducing some sort of regularization, such as a finite-volume interpretation as employed here and in previous works [36]. One also needs to be careful about the cell size choice and possible nonphysical effects such as negative densities, which may arise from the Gaussian processes. In Sect. 3 we propose a finite-volume interpretation of Eq. (5) which correctly overcomes these issues.

Previous numerical methodologies for FH have been focused on the LLNS equations for the density and momentum, and the energy equation for the temperature if the systems are non-isothermal. In comparison, the overdamped FDDFT allows us to obtain the density field solving a single equation with stochastic fluxes for isothermal systems. One of the first works on this regard is by Garcia et al. [37], where a simple finite-difference scheme to treat the numerical fluxes of the SPDE is constructed. Further works by Bell et al. [38,39] provide an explicit Eulerian discretization of the LLNS equations combined with a third-order Runge-Kutta method with the objective of adequately reproducing the fluctuations in density, energy and momentum. Donev and co-workers [36] exploited the structure factor (equilibrium fluctuation spectrum) to construct finite-volume schemes to solve the LLNS which then allows one to study the accuracy for a given discretization at long wavelengths. They also proposed a Petrov-Galerkin finite-element discretization of non-linear stochastic diffusion equations embedded with prototypical free energy functionals, such as the Ginzburg-Landau free energy [40]. However, in order to obtain analytical forms of the structure factors used to assess the performance of the scheme, the study focused on systems at equilibrium (i.e. without density discontinuities), at supercritical temperatures (to avoid phase transition phenomena), and without any external potential. Similarly, methods to solve FH via staggered grids have been constructed [41]. Other works have proposed numerical schemes based on temporal integrators that are implicit-explicit predictor-corrector [42] or two-level leapfrog [43]. Additionally, hybrid schemes have been developed to couple LLNS with MD [44, 45, 46] or with MC [47, 48] simulations of complex fluid systems. Moreover, the LLNS have also been solved to tackle reactive multi-species fluid mixtures [49]. Further works have developed numerical schemes for particular applications of the overdamped FDDFT in Eq. (5). Specifically, Refs [35, 50] developed numerical methods for reaction-diffusion equations obtained by adding appropriate reaction terms to Eq. (5) equipped with the ideal-gas free-energy functional.

The works just mentioned have contributed to a better understanding of the effects of thermal fluctuations in complex fluid systems. Nevertheless, an efficient and systematic numerical methodology to solve Eq. (5) equipped with a general free-energy functional has not yet been developed. Such a methodology would allow for the simulation and scrutiny of a wide range of non-equilibrium phenomena which can be studied within the framework of FDDFT. Relevant examples of these physical phenomena include dynamic evolution of confined systems and energy-barrier crossing transitions, such as nucleation.

In this work we introduce a finite-volume method to solve general stochastic gradient flow equations with the structure of Eq. (5) for FDDFT. The main advantages of finite-volume schemes are the conservation of the total mass of the system and the flexibility to simulate complex geometries. The main contributions of this work can be summarized as follows: 
- providing a space discretization scheme able to deal with fluctuations at discontinuous density profiles. We discretize the deterministic fluxes based on a hybrid approach which takes advantage of both central and upwind schemes.

- overcoming the commonplace challenge of preserving non-negative densities in the presence of noise, a Brownian bridge technique is adopted. Despite previous approaches employing artificial limiters [35], our technique ensures density positivity without altering the Gaussian distribution of the stochastic field.

- developing a methodology to simulate a family of free-energy functionals, modelling different physical systems. First, we study temporal and spatial correlations, and structure factor of ideal gas at equilibrium, comparing the results of our finite-volume solver with both MD and theoretical results. Then, we examine the out-of-equilibrium evolution of an ideal gas in a double-well external potential. Subsequently, we simulate homogeneous nucleation kinetics of a fluid consisting of particles interacting through a Lennard-Jones (LJ)like potential. Providing initial uniform densities corresponding to metastable vapour conditions, we study the phase-transition of the system and compare the results with the mean-field phase diagram.

- implementing and testing families of implicit-explicit Euler and Milsten time integrators, together with a weak second-order Runge-Kutta scheme.

- getting insights into the free energy decay for stochastic gradient flow equations (see for instance Figs 8(d) and 10(b)). The decay of free energy is an important feature of deterministic gradient flow equations. However, in stochastic gradient flow equations, the free energy decay is guaranteed only in the weak noise limit as discussed at page 4, Eqs (8)-(9).

In Sect. 2, we present the model equation to simulate and outline its main properties. In Sect. 3, we discuss the numerical methodology of our finite-volume scheme, including flux discretization, time integrators, adaptive time step to preserve density positivity and boundary conditions. Several applications to illustrate the validity of our methodology are presented in Sects 4. Finally, a summary and conclusions are offered in Sect. 5.

\section{Governing equations and related properties}

Our starting point is the following general SPDE based on the overdamped FDDFT in Eq. (5) with $\gamma=1$ and $m=1$,

$$
\left\{\begin{array}{l}
\partial_{t} \rho(\boldsymbol{r} ; t)=\nabla_{\mathbf{r}} \cdot\left[\rho(\boldsymbol{r} ; t) \boldsymbol{\nabla}_{\mathbf{r}} \frac{\delta \mathcal{E}[\rho]}{\delta \rho(\boldsymbol{r} ; t)}\right]+\nabla_{\mathbf{r}} \cdot[\sqrt{\rho(\boldsymbol{r} ; t) / \beta} \boldsymbol{W}(\boldsymbol{r} ; t)] \quad \mathbf{r} \in \mathbb{R}^{d}, t>0, \\
\rho(\mathbf{r} ; 0)=\rho_{0}(\mathbf{r}),
\end{array}\right.
$$

where $\mathcal{E}[\rho]$ denotes the free energy of the system given by

$$
\mathcal{E}[\rho]=\int_{\mathbb{R}^{d}} f(\rho) d \mathbf{r}+\int_{\mathbb{R}^{d}} V(\mathbf{r}) \rho d \mathbf{r}+\frac{1}{2} \int_{\mathbb{R}^{d}} g(K(\mathbf{r}) * \rho(\mathbf{r})) \rho(\mathbf{r}) d \mathbf{r},
$$

with $f(\rho)$ describing the dependency of the free energy $\mathcal{E}[\rho]$ on the local density field $\rho, V(\mathbf{r})$ accounting for the effects of external potentials, $g$ denoting a function depending on the convolution of $\rho(\mathbf{r})$ with the symmetric kernel $K(\mathbf{r})$ accounting for the interparticle potential. For simplicity, we introduce the constant $\beta$, defined as $\beta=\left(k_{B} T\right)^{-1}$.

The mean-field limit of Eq. (6) in which no stochastic flux is present has received a great deal of attention in the context of gradient flows. As discussed in Ref. [11], in the weak noise limit, the most-likely path followed by the system minimizes the Lagrangian defined as $\mathcal{L}=\left\|\partial_{t} \rho-\boldsymbol{\nabla}_{\boldsymbol{r}} \cdot\left(\rho(\boldsymbol{r} ; t) \boldsymbol{\nabla}_{\boldsymbol{r}} \frac{\delta \mathcal{E}[\rho]}{\delta \rho}\right)\right\|_{\left(\sigma \sigma^{*}\right)^{-1}}$, where $\sigma$ is the operator acting on the noise $\boldsymbol{W}(\boldsymbol{r} ; t)$. Thus, the most-likely solution $\langle\rho\rangle(\boldsymbol{r} ; t)$ satisfies

$$
\partial_{t}\langle\rho\rangle(\boldsymbol{r} ; t)=\boldsymbol{\nabla}_{\boldsymbol{r}} \cdot\left(\langle\rho\rangle \boldsymbol{\nabla}_{\boldsymbol{r}} \frac{\delta \mathcal{E}[\langle\rho\rangle]}{\delta\langle\rho\rangle}\right) .
$$

Equation (8) is a generalized diffusion equation, which results in the heat equation if an ideal gas free energy is selected. It has been widely employed not only in the framework of DDFT [14, 15, 25], but also to model thin 
liquid films stochastic dynamics[51]. It has the structure of a gradient flows in the Wasserstein metric [52, 53] with applications in a variety of contexts such as granular media [34], materials science and biological swarming $[34,54,55]$. The fundamental property of Eq. (8) is that the free energy (7) is minimized following the decay rate $[34,56,57]$

$$
\frac{d}{d t} \mathcal{E}[\langle\rho\rangle]=-\int_{\mathbb{R}^{d}}\langle\rho\rangle\left|\frac{\delta \mathcal{E}[\langle\rho\rangle]}{\delta\langle\rho\rangle}\right|^{2} d \mathbf{r},
$$

where the variation of the free energy $\mathcal{E}[\rho]$ with respect to the density $\rho$ in the case of (7) satisfies

$$
\frac{\delta \mathcal{E}[\rho]}{\delta \rho}=f^{\prime}(\rho)+V(\mathbf{r})+K *\left(g^{\prime}(K * \rho) \rho\right)+g(K * \rho) .
$$

The decay rate in Eq. 9 is not satisfied by the stochastic gradient flow in Eq. (6), where punctual increases in the free energy during the dynamical evolution can take place. Precisely, these jumps allow the system to overcome energy barriers leading to phenomena such as phase transitions.

\subsection{Structure factor}

The structure factor is a quantity of interest in many fields, including FH [36] as noted earlier and capillary wave theory [58, 59]. As shown in previous works [36, 35], the structure factor represents an important measure of the stochastic properties of the system and it can be experimentally accessible. It is valuable not only to study the stability of the numerical integrator, but also to compare different schemes, as it will be shown in Sect.3. Here we derive an expression of the structure factor from the linearized FDDFT. If we consider a periodic domain of volume $V$, the spatial Fourier transform of the density is given by

$$
\hat{\rho}_{\lambda}=\frac{1}{V} \int_{V} \rho(\mathbf{r}, t) e^{-i \lambda \cdot \mathbf{r}} d \mathbf{r} .
$$

The structure factor is defined as the variance of the Fourier transform of the density fluctuations,

$$
S(\lambda)=V\left\langle\delta \hat{\rho}_{\lambda} \delta \hat{\rho}_{\lambda}^{*}\right\rangle,
$$

where $\delta \hat{\rho}_{\lambda}=\hat{\rho}_{\lambda}-\left\langle\hat{\rho}_{\lambda}\right\rangle$, and $\hat{\rho}_{\lambda}^{*}$ denotes the complex conjugate of $\hat{\rho}_{\lambda}$.

For uniform systems, Eq. (6) can be formally linearized around its most-likely solution $\langle\rho\rangle$ by means of the central limit theorem, giving

$$
\partial_{t} \rho(\boldsymbol{r} ; t)=\boldsymbol{\nabla}_{\boldsymbol{r}} \cdot\left(\rho(\boldsymbol{r} ; t) \boldsymbol{\nabla}_{\boldsymbol{r}} \frac{\delta \mathcal{E}[\rho]}{\delta \rho}\right)+\sqrt{\langle\rho\rangle / \beta} \boldsymbol{\nabla}_{\boldsymbol{r}} \cdot \boldsymbol{W}(\boldsymbol{r} ; t) .
$$

Taking the Fourier transform of the difference between Eq. (13) and Eq. (8), one obtains

$$
\partial_{t} \delta \hat{\rho}(\lambda)=i \lambda \cdot\left\{\mathcal{T}\left(\rho(\boldsymbol{r} ; t) \boldsymbol{\nabla}_{\boldsymbol{r}} \frac{\delta \mathcal{E}[\rho]}{\delta \rho}\right)-\mathcal{T}\left(\langle\rho\rangle \boldsymbol{\nabla}_{\boldsymbol{r}} \frac{\delta \mathcal{E}[\langle\rho\rangle]}{\delta\langle\rho\rangle}\right)\right\}+i \boldsymbol{\lambda} \cdot \sqrt{\langle\rho\rangle / \beta} \hat{\boldsymbol{W}}(\boldsymbol{\lambda})
$$

where $\mathcal{T}$ denotes the Fourier transform. If the free energy functional terms in the Fourier space can be expanded at first order around their mean value as

$$
\mathcal{T}\left(\rho(\boldsymbol{r} ; t) \nabla_{\boldsymbol{r}} \frac{\delta \mathcal{E}[\rho]}{\delta \rho}\right) \sim \mathcal{T}\left(\langle\rho\rangle \boldsymbol{\nabla}_{\boldsymbol{r}} \frac{\delta \mathcal{E}[\langle\rho\rangle]}{\delta\langle\rho\rangle}\right)+\frac{\partial \mathcal{T}\left[\rho(\boldsymbol{r} ; t) \boldsymbol{\nabla}_{\boldsymbol{r}} \frac{\delta \mathcal{E}[\rho]}{\delta \rho}\right]}{\partial \hat{\rho}_{\lambda}} \delta \hat{\rho}_{\lambda}+O\left(\delta \hat{\rho}_{\lambda}\right),
$$

then Eq. 14 yields

$$
\partial_{t} \delta \hat{\rho}_{\lambda}=i \lambda \cdot \frac{\partial \mathcal{T}\left[\rho(\boldsymbol{r} ; t) \nabla_{\boldsymbol{r}} \frac{\delta \mathcal{E}[\rho]}{\delta \rho}\right]}{\partial \hat{\rho}_{\lambda}} \delta \hat{\rho}_{\lambda}+i \lambda \cdot \sqrt{\langle\rho\rangle / \beta} \hat{\boldsymbol{W}}(\lambda) .
$$

Since the above equation has the form of an Ornstein-Uhlenbeck process, the structure factor can be computed as its variance:

$$
S(\lambda)=\frac{2(i \lambda \sqrt{\langle\rho\rangle / \beta})^{2}}{2 i \lambda \frac{\partial \mathcal{T}\left[\rho(\boldsymbol{r} ; t) \nabla_{r} \frac{\delta \delta[\rho]}{\delta \rho}\right]}{\partial \hat{\rho}_{\lambda}}}=\frac{i \lambda\langle\rho\rangle / \beta}{\frac{\partial \mathcal{T}\left[\rho(\boldsymbol{r} ; t) \nabla_{r} \frac{\delta \delta \rho \rho]}{\delta \rho}\right]}{\partial \hat{\rho}_{\lambda}}}
$$


For example, in the case of an ideal gas without external potential, $\frac{\delta \mathcal{E}[\rho]}{\delta \rho}=\log \rho$, the structure factor is given by the well-known expression [35]:

$$
S(\lambda)=\frac{i \lambda\langle\rho\rangle / \beta}{\frac{\partial \mathcal{T}\left[\rho(\boldsymbol{r} ; t) \nabla_{r} \log \rho(\boldsymbol{r}, t)\right]}{\partial \hat{\rho}_{\lambda}}}=\frac{i \lambda\langle\rho\rangle / \beta}{\frac{\partial \mathcal{T}\left[\nabla_{r} \rho(\boldsymbol{r}, t)\right]}{\partial \hat{\rho}_{\lambda}}}=\frac{i \lambda\langle\rho\rangle / \beta}{\frac{\partial[i \lambda \hat{\rho}]}{\partial \hat{\rho}(\lambda)}}=\langle\rho\rangle / \beta .
$$

\section{Numerical methods}

The one-dimensional version of Eq. (6) can be written as

$$
\partial_{t} \rho=\partial_{x} F_{d}(\rho)+\partial_{x} F_{s}(\rho, \mathcal{W}),
$$

where $F_{d}$ and $F_{s}$ denote the deterministic and stochastic fluxes, respectively,

$$
F_{d}=\rho \partial_{x} \frac{\delta \mathcal{E}[\rho]}{\delta \rho}, \quad F_{s}=\sqrt{\rho / \beta} \mathcal{W} .
$$

The finite-volume formulation of Eq. (19) is obtained by dividing the domain into grid cells $C_{j}=\left[x_{j-\frac{1}{2}}, x_{j+\frac{1}{2}}\right]$, each one assumed to have the same length $\Delta x=x_{j+1 / 2}-x_{j-1 / 2}$, and then approximating in each of them the cell average of $\rho$ defined as

$$
\bar{\rho}_{j}(t)=\frac{1}{\Delta x} \int_{x_{j-1 / 2}}^{x_{j+1 / 2}} \rho(x, t) d x .
$$

Subsequently, one has to integrate (19) spatially over each cell and apply the Gauss divergence theorem, leading eventually to the semi-discrete equation for the temporal evolution of the cell average density,

$$
\frac{d \bar{\rho}_{j}}{d t}=\frac{F_{d, j+1 / 2}-F_{d, j-1 / 2}}{\Delta x}+\frac{F_{s, j+1 / 2}-F_{s, j-1 / 2}}{\Delta x},
$$

where $F_{d, j+1 / 2}$ and $F_{s, j+1 / 2}$ denote the deterministic and stochastic fluxes (20) evaluated at the boundary $x_{j+1 / 2}$. The separation of the physical flux into deterministic and stochastic parts has been effectively applied in previous studies $[38,36]$, noting though that some studies do consider a unique flux combining the deterministic and stochastic terms [60]. Here we treat them separately. In the following subsections, we proceed to develop in detail the methodology of our finite-volume scheme.

\subsection{Deterministic flux}

The deterministic flux is evaluated by employing a hybrid method, which adopts a central or upwind approximation depending on the relative local total variation of the density. This is a classical technique in deterministic fluid dynamics to construct high-resolution and oscillation-free schemes [61]. On the one hand, central high-order and non-diffusive schemes are applied wherever no sharp gradients of the density are found. On the other hand, a diffusive upwind scheme is employed in those regions of the domain with density gradients, in order to prevent the spurious oscillations from central high-order schemes.

Previous works in the field of $\mathrm{FH}[38,36,35]$ approximate the deterministic flux with a simple second-order central difference approach, even though high-order differences are also proposed but not implemented [36]. Our motivation to propose a hybrid approach is precisely aimed to avoid possible spurious oscillations. The previous literature is mainly focused on FH with $f^{\prime}(\rho)=\log \rho$ in Eq. (10), resulting in a deterministic flux of the form $\partial_{x} F_{d}(\rho)=\partial_{x x} \rho$. The treatment of this Laplacian with a central approximation works well for the cases presented in the literature, but as it is shown later in Fig. 1, it can cause spurious oscillations for some solutions.

In the case of Eq. (19), the stochastic flux leads to non-smooth density. Because of this the proposed hybrid scheme compares the local gradient in the density with the neighbouring gradients. When the local gradient is large in comparison, an upwind approximation is chosen. If not, the central approximation prevails. As a result, our proposed hybrid scheme for the deterministic flux satisfies

$$
F_{d, j+1 / 2}=\left(1-\phi\left(r_{j+1 / 2}\right)\right) F_{d, j+1 / 2}^{c}+\phi\left(r_{j+1 / 2}\right) F_{d, j+1 / 2}^{u},
$$


where $\phi\left(r_{j+1 / 2}\right)$ is a flux limiter with a threshold parameter $k$, defined as

$$
\phi\left(r_{j+1 / 2}\right)= \begin{cases}0, & \text { if } r_{j+1 / 2} \leq k, \\ 1, & \text { if } r_{j+1 / 2}>k,\end{cases}
$$

and $r_{j+1 / 2}$ is a quotient measuring the relative local variation of the density,

$$
r_{j+1 / 2}=\frac{\left|\rho_{j+1}-\rho_{j}\right|}{\sum_{l=-w}^{w}\left|\rho_{l+1}-\rho_{l}\right|},
$$

with $w$ indicating the number of neighbouring cell used to compute the total variation. A value $w=5$ is employed in the numerical experiments of this work, since it gives a good compromise between conservation of local information and effects of the fluctuations.

The threshold parameter $k$ plays a key role and has to be carefully selected. When $k$ is small, the diffusive upwind scheme is chosen more frequently, leading to diffusive behaviour which affects the structure factor and the correlations. On the contrary, when $k$ is large, the central scheme will be predominant, and spurious oscillations may be created. Fig. 1 provides a numerical example to choose an adequate value for $k$.

Firstly, Figs $1(\mathrm{a}-\mathrm{b})$ are obtained by simulating (19) with a free energy satisfying $\delta \mathcal{E} / \delta \rho=\log \rho+0.1 x$. The initial density profile has two discontinuities as shown in Fig. 1 (a). Under these conditions, the numerical solution evolves as a diffusive travelling wave, but the two discontinuities in the initial density trigger spurious oscillations. The oscillations diminish by reducing $k$ (for $k=0$, which corresponds to only upwind flux, the diffusion eliminates the oscillations). However, a low value of $k$ critically dampens the variance, due to the diffusive nature of the upwind flux, as it is noticed from Fig. 1 (b).

Secondly, Fig. 1 (c) is obtained from simulating (19) with a free energy satisfying $\delta \mathcal{E} / \delta \rho=\log \rho$ and starting from an equilibrium density profile. For this case, the theoretical value of the structure factor is known and is given by (18), meaning that the dampening behaviour of the upwind scheme could be directly evaluated from Fig. 1 (c). It is possible to notice again how the upwind scheme dampens the statistical properties of the system due to the numerical diffusion. As a result, an intermediate value of $k$ needs to be taken in order to find a balance between both numerical flaws. The compromising value is chosen to be $k=3$.

(a)

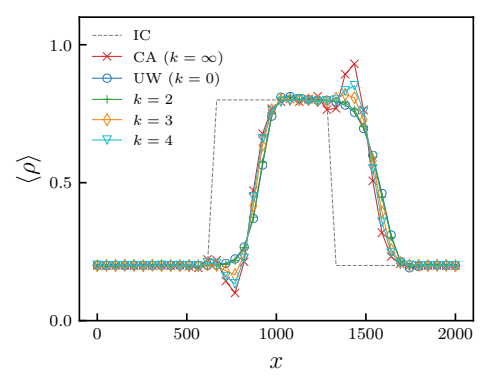

(b)

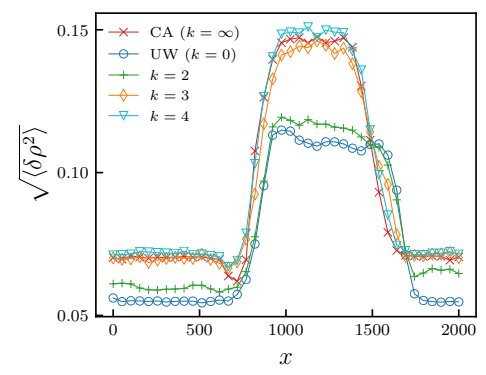

(c)

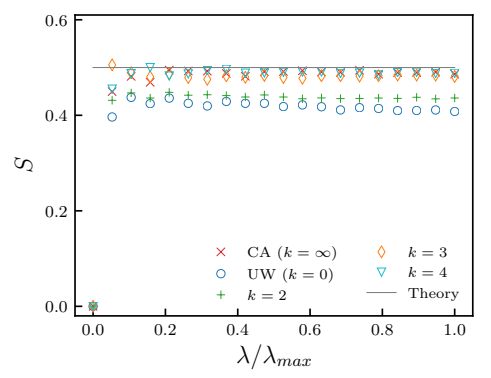

Fig. 1: (a) Mean density and (b) standard deviation for a moving and diffusing initial step function evolving under (19), with $\delta \mathcal{E} / \delta \rho=\log \rho+u_{0} x$ with $u_{0}=0.1$. For clarity, the structure factor for a uniform system is also reported in (c). IC: initial condition, CA: central approximation $(k=\infty)$, UW: upwind approximation $(k=0)$. Further values of $k$ are depicted to evaluate the spurious oscillations in the density and the artificial fluctuation dampening in case of both inhomogeneous (a-b) and homogeneous (c) systems. In what follows, we adopt a scheme with $k=3$, since it gives the compromise between accuracy in sharp density profile and fluctuations amplitude. The stochastic term is discretized according to Eq. (37).

After selecting the adequate value of $k$, we proceed to the detailed construction of the central and upwind deterministic fluxes in (23):

a) Upwind approximation of the deterministic flux: it is constructed as proposed in [62], where a first- and secondorder finite-volume method for nonlinear equations with gradient flow structure is constructed. The equations treated in [62] have the form (19) without the white noise $\mathcal{W}$. The authors propose to firstly reconstruct the 
density profile in each cell $C_{j}$ as a constant profile for the first-order scheme, or as a linear profile for the second-order scheme,

$$
\widetilde{\rho}_{j}(x)=\left\{\begin{array}{l}
\bar{\rho}_{j}, \quad x \in C_{j}, \quad \text { for the first-order scheme } \\
\bar{\rho}_{j}+\left(\rho_{x}\right)_{j}\left(x-x_{j}\right), \quad x \in C_{j}, \quad \text { for the second-order scheme }
\end{array}\right.
$$

so that the east and the west density values $\rho_{j}^{E}$ and $\rho_{j}^{W}$ at the cell interfaces $x_{j+\frac{1}{2}}$ and $x_{j-\frac{1}{3}}$, respectively, are approximated as

$$
\begin{aligned}
& \rho_{j}^{E}=\bar{\rho}_{j}+\frac{\Delta x}{2}\left(\rho_{x}\right)_{j}, \\
& \rho_{j}^{W}=\bar{\rho}_{j}-\frac{\Delta x}{2}\left(\rho_{x}\right)_{j} .
\end{aligned}
$$

The numerical derivatives $\left(\rho_{x}\right)_{j}$ at every cell $C_{j}$ are computed by means of an adaptive procedure which ensures that the point values (26) are second-order and non-negative. This procedure initially takes centred approximations of the form $\left(\rho_{x}\right)_{j}=\left(\bar{\rho}_{j+1}-\bar{\rho}_{j-1}\right) /(2 \Delta x)$. If it then happens that $\rho_{j}^{E}<0$ or $\rho_{j}^{W}<0$, the scheme employs a minmod limiter which ensures that the reconstructed values are non-negative as far as the cell averages $\bar{\rho}_{j}$ are non-negative,

$$
\left(\rho_{x}\right)_{j}=\operatorname{minmod}\left(\theta \frac{\bar{\rho}_{j+1}-\bar{\rho}_{j}}{\Delta x}, \frac{\bar{\rho}_{j+1}-\bar{\rho}_{j-1}}{2 \Delta x}, \theta \frac{\bar{\rho}_{j}-\bar{\rho}_{j-1}}{\Delta x}\right),
$$

where

$$
\operatorname{minmod}\left(z_{1}, z_{2}, \ldots\right)=\left\{\begin{array}{lr}
\min \left(z_{1}, z_{2}, \ldots\right), & \text { if } z_{i}>0 \quad \forall i, \\
\max \left(z_{1}, z_{2}, \ldots\right), & \text { if } z_{i}<0 \quad \forall i, \\
0, & \text { otherwise. }
\end{array}\right.
$$

The parameter $\theta$ controls the numerical viscosity and it is taken to be $\theta=2$, as in Ref. [62].

After completing the density reconstruction, the deterministic flux $F_{d, j+1 / 2}^{u}$ is evaluated with an upwind scheme as

$$
F_{d, j+1 / 2}^{u}=u_{j+1 / 2}^{+} \rho_{j}^{E}+u_{j+1 / 2}^{-} \rho_{j+1}^{W},
$$

where $u_{j+1 / 2}$ are discrete values computed from the central difference

$$
u_{j+1 / 2}=-\frac{\left(\frac{\delta \mathcal{E}}{\delta \rho}\right)_{j+1}-\left(\frac{\delta \mathcal{E}}{\delta \rho}\right)_{j}}{\Delta x} .
$$

The upwind formulation of the deterministic flux (28) is then accomplished by taking

$$
u_{j+1 / 2}^{+}=\max \left(u_{j+1 / 2}, 0\right) \text { and } u_{j+1 / 2}^{-}=\min \left(u_{j+1 / 2}, 0\right) .
$$

Finally, the discrete variation of the free energy with respect to the density $\left(\frac{\delta \mathcal{E}}{\delta \rho}\right)_{j}$ is computed from (10), in the case $g(s)=s$, as

$$
\left(\frac{\delta \mathcal{E}}{\delta \rho}\right)_{j}=\Delta x \sum_{i} K\left(x_{j}-x_{i}\right) \rho_{i}+F\left(\rho_{j}\right)+V\left(x_{j}\right) .
$$

For general nonlinearities $g(s)$ a similar treatment is performed.

b) Central approximation for the deterministic flux: this is the main strategy to treat the FH deterministic flux in the literature $[38,36,35]$. In our case, given the generality of the free energy in (7), we propose to evaluate the central deterministic flux as

$$
F_{d, j+1 / 2}^{c}=u_{j+1 / 2} \rho_{j+1 / 2},
$$


where $u_{j+1 / 2}$ is computed as in (29), with the discrete variation of the free energy satisfying (31), and $\rho_{j+1 / 2}$ is taken as the averaged from the adjacent cells,

$$
\rho_{j+1 / 2}=\frac{\bar{\rho}_{j}+\bar{\rho}_{j+1}}{2} .
$$

The classical hybrid schemes employ a high-order approximation for the central approximation of the deterministic flux. For this work, however, we just consider the low-order differences (29) and (33), given that the presence of the stochastic flux limits the spatial order of accuracy. Previous works in the literature also propose this low-order central differences $[38,36,35]$.

\subsection{Stochastic flux}

The evaluation of the stochastic flux (20) must be done carefully since the divergence of the white noise $\mathcal{W}$ cannot be evaluated pointwise in time and space. This problem is typically overcome by evaluating the noise in the cell by means of a spatiotemporal average, following the work in [36] and subsequently employed by Donev and collaborators in $[63,49,35]$,

$$
\overline{\mathcal{W}}_{j}=\frac{1}{\Delta x \Delta t} \int_{t}^{t+\Delta t} \int_{x_{j-\frac{1}{2}}}^{x_{j+\frac{1}{2}}} \mathcal{W}(x, t) d x d t,
$$

which, by definition of the white noise, is equal to a normal distribution with zero mean and variance $(\Delta x \Delta t)^{-1}$, so that

$$
\overline{\mathcal{W}}_{j}=\mathcal{N}(0,1) / \sqrt{\Delta x \Delta t}
$$

Several approximations for the stochastic flux have been put forward in the literature $[38,35]$. They rely on computing the stochastic flux directly at the interfaces using a random number generator, and we refer the reader to [36] for more details about this approach. In this work, however, we aim to employ the spatiotemporal cell average in Eq. (34) to compute the stochastic fluxes at the interfaces. We are inspired by the literature on numerical methods for hyperbolic problems where it is common to evaluate fluxes in a central or upwind fashion. Of course, here we are not aiming to achieve a higher accuracy at the interface, given that the cell averages are sampled from a distribution. Here we test the following four different approximations for the stochastic flux, which are compared in Sect. 4:

(a) Forward approximation of the form

$$
F_{s, j+1 / 2}=\left(\sqrt{\frac{\rho}{\beta}} \mathcal{W}\right)_{j+1 / 2}=\sqrt{\frac{\rho_{j}}{\beta}} \overline{\mathcal{W}}_{j}
$$

(b) Linear approximation of the form

$$
F_{s, j+1 / 2}=\left(\sqrt{\frac{\rho}{\beta}} \mathcal{W}\right)_{j+1 / 2}=\sqrt{\frac{\rho_{j+1 / 2}}{\beta}} \mathcal{W}_{j+1 / 2},
$$

where

$$
\rho_{j+1 / 2}=\frac{\bar{\rho}_{j}+\bar{\rho}_{j+1}}{2}, \quad \mathcal{W}_{j+1 / 2}=\frac{\overline{\mathcal{W}}_{j}+\overline{\mathcal{W}}_{j+1}}{2} .
$$

(c) Parabolic approximation of the form

$$
F_{s, j+1 / 2}=\left(\sqrt{\frac{\rho}{\beta}} \mathcal{W}\right)_{j+1 / 2}=\sqrt{\frac{\rho_{j+1 / 2}}{\beta}} \mathcal{W}_{j+1 / 2},
$$

where

$$
\begin{gathered}
\rho_{j+1 / 2}=\alpha_{1}\left(\bar{\rho}_{j-1}+\bar{\rho}_{j+2}\right)+\alpha_{2}\left(\bar{\rho}_{j}+\bar{\rho}_{j+1}\right), \\
\mathcal{W}_{j+1 / 2}=\alpha_{1}\left(\overline{\mathcal{W}}_{j-1}+\overline{\mathcal{W}}_{j+2}\right)+\alpha_{2}\left(\overline{\mathcal{W}}_{j}+\overline{\mathcal{W}}_{j+1}\right), \\
\alpha_{1}=(1-\sqrt{3}) / 4, \quad \alpha_{2}=(1+\sqrt{3}) / 4 .
\end{gathered}
$$

The coefficients $\alpha_{1}$ and $\alpha_{2}$ are selected as in [38], with the objective of preserving both the average and the variance in each time step. 
(d) Upwind approximation, where $\mathcal{W}_{j}$ is taken as the stochastic velocity, so that a similar expression to the deterministic flux in (28) is taken,

$$
F_{s, j+1 / 2}=\left(\sqrt{\frac{\rho}{\beta}} \mathcal{W}\right)_{j+1 / 2}=\sqrt{\frac{\rho_{j}^{E}}{\beta}} W_{j+1 / 2}^{+}+\sqrt{\frac{\rho_{j+1}^{W}}{\beta}} W_{j+1 / 2}^{-},
$$

where

$$
\mathcal{W}_{j+1 / 2}^{+}=\max \left(\mathcal{W}_{j+1 / 2}, 0\right), \quad \mathcal{W}_{j+1 / 2}^{-}=\min \left(\mathcal{W}_{j+1 / 2}, 0\right),
$$

and $\mathcal{W}_{j+1 / 2}=\left(\overline{\mathcal{W}}_{j}+\overline{\mathcal{W}}_{j+1}\right) / 2$. The east and west density values $\rho_{j}^{E}$ and $\rho_{j}^{W}$ are computed as in the deterministic flux, either with a first- or second-order reconstruction (26).

\subsection{Stochastic time integrators}

The derivation of the temporal integrators to advance in time the semidiscrete equation (22) is accomplished by the equation

$$
d \bar{\rho}(t)=\boldsymbol{\mu}(\overline{\boldsymbol{\rho}}(t)) d t+\boldsymbol{\sigma}(\overline{\boldsymbol{\rho}}(t)) \overline{\boldsymbol{W}} d t
$$

where the vectors $\bar{\rho}(t)$ and $\overline{\boldsymbol{W}}$ contain the cell averages defined in (21) and (34), respectively, so that $\overline{\boldsymbol{\rho}}(t)=$ $\left(\bar{\rho}_{1}(t), \bar{\rho}_{2}(t), \ldots, \bar{\rho}_{n}(t)\right)$ and $\overline{\boldsymbol{W}}(t)=\left(\overline{\mathcal{W}}_{1}(t), \overline{\mathcal{W}}_{2}(t), \ldots, \overline{\mathcal{W}}_{n}(t)\right)$. The vector $\boldsymbol{\mu}(\overline{\boldsymbol{\rho}}(t))$ and the matrix $\boldsymbol{\sigma}(\overline{\boldsymbol{\rho}}(t))$ depend on the density cell averages $\bar{\rho}(t)$ and their structures vary depending on the choice of the deterministic and stochastic fluxes, respectively.

From Eq. (43) one can employ Itô's lemma to approximate the two functions $\boldsymbol{\mu}(\overline{\boldsymbol{\rho}}(t))$ and $\boldsymbol{\sigma}(\overline{\boldsymbol{\rho}}(t))$. After integrating in time then one obtains the Taylor expansion of the stochastic process. Truncating this expansion with an error $O\left(\Delta t^{1 / 2}\right)$ and integrating between $t$ and $t+\Delta t$, one can derive the following family of implicit-explicit Euler-Maruyama integrators [64], whose component-wise form satisfies

$$
\bar{\rho}_{j}(t+\Delta t)=\bar{\rho}_{j}(t)+\left[(1-\theta) \mu_{j}(\overline{\boldsymbol{\rho}}(t))+\theta \mu_{j}(\overline{\boldsymbol{\rho}}(t+\Delta t))\right] \Delta t+\sum_{k=1}^{n} \sigma_{j k}\left(\overline{\boldsymbol{\rho}}_{(t))} \bar{W}_{k}(t) \Delta t .\right.
$$

The parameter $\theta$ allows us to have an explicit $(\theta=0)$, implicit $(\theta=1)$ or semi-implicit $(\theta=0.5)$ temporal integrator. Euler-Maruyama is the highest order integrator for which no multiple stochastic integrals have to be computed, but it has only 0.5 strong order of convergence.

Keeping in the expansion all the terms up to $O(\Delta t)$, one obtains a derivative-free family of implicit-explicit Milstein integrators with strong order 1.0 and weak order 0.5 [64]. The component-wise version of this scheme is

$$
\begin{aligned}
\bar{\rho}_{j}(t+\Delta t)= & \bar{\rho}_{j}(t)+\left[(1-\theta) \mu_{j}(\overline{\boldsymbol{\rho}}(t))+\theta \mu_{j}(\overline{\boldsymbol{\rho}}(t+\Delta t))\right] \Delta t+\sum_{k=1}^{n} \sigma_{j k}(\overline{\boldsymbol{\rho}}(t)) \bar{W}_{k}(t) \Delta t \\
& +\frac{1}{\sqrt{\Delta t}} \sum_{l, m=1}^{n}\left[\sigma_{j m}\left(\Upsilon_{l}(t)\right)-\sigma_{j l}(\overline{\boldsymbol{\rho}}(t))\right] I_{l, m}(t)
\end{aligned}
$$

where the $l$-th row of the matrix $\Upsilon$ is defined as

$$
\Upsilon_{l}(t)=\overline{\boldsymbol{\rho}}(t)+\boldsymbol{\mu}(\overline{\boldsymbol{\rho}}(t)) \Delta t+\boldsymbol{\sigma}_{l}(\overline{\boldsymbol{\rho}}(t)) \sqrt{\Delta t},
$$

and multiple stochastic integrals $I_{l, m}(t)=\int_{t}^{t+\Delta t} \mathcal{W}^{l} \mathcal{W}^{m} d t$, where $\mathcal{W}_{l}$ and $\mathcal{W}_{m}$ are two white noises. These integrals do not have a simple analytical solutions, thus are approximated as function of the white noise cells average in Eq. (34) as [65]:

$I_{l, m}(t)= \begin{cases}\frac{1}{2}\left[\left(\bar{W}^{l}\right)^{2}-1\right] \Delta t & \text { if } \quad l=m, \\ \frac{\Delta t}{2} \bar{W}^{l} \bar{W}^{m}+\sqrt{k_{p} \Delta t}\left(\varphi_{l} \bar{W}^{m}-\varphi_{m} \bar{W}^{l}\right)+\sum_{r=1}^{p} \frac{1}{2 \pi r}\left[\zeta_{l r}\left(\sqrt{2} \bar{W}^{m} \sqrt{\Delta t}+\eta_{m}\right)-\zeta_{m r}\left(\sqrt{2} \bar{W}^{l} \sqrt{\Delta t}+\eta_{l}\right)\right] & \text { otherwise, }\end{cases}$ 
where $\varphi_{l}, \zeta_{l r}$ and $\eta_{m}$ are pairwise independent variables with distribution $\mathcal{N}(0, \Delta t)$ and $k_{p}$ is given by

$$
k_{p}=\frac{1}{12}-\frac{1}{2 \pi^{2}} \sum_{1}^{p} \frac{1}{r^{2}} .
$$

The value $p$ determines the accuracy of the multiple stochastic integral approximation, and then of the scheme. A value of $p=k / \Delta t$ for some constant $k$ is enough to preserve the accuracy of the scheme [64].

Stochastic time integration schemes of higher strong order have also been proposed in the literature [64]. However, these schemes are very computationally expensive due to the presence of high-order multiple stochastic integrals to be solved. Moreover, in many physical applications, the convergence in probability, also called weak convergence, is more relevant than the strong convergence. For this reason, as last time integration scheme we will study the following explicit weak order 2.0 Runge-Kutta scheme:

$$
\begin{aligned}
& \widetilde{\rho}_{j}(t+\Delta t)=\bar{\rho}_{j}(t)+\frac{1}{2}\left[\mu_{j}(\Upsilon(t))+\mu_{j}(\overline{\boldsymbol{\rho}}(t))\right] \Delta t+\Phi(t), \\
& \bar{\rho}_{j}(t+\Delta t)=\bar{\rho}_{j}(t)+\frac{1}{2}\left[\mu_{j}(\widetilde{\boldsymbol{\rho}}(t+\Delta t))+\mu_{j}(\overline{\boldsymbol{\rho}}(t))\right] \Delta t+\Phi(t),
\end{aligned}
$$

where the vector $\Phi(t)$ has components:

$$
\begin{aligned}
\Phi_{j}(t)= & \frac{1}{4} \sum_{l=1}^{n}\left[\sigma_{l j}\left(\boldsymbol{\Lambda}_{l+}(t)\right)+\sigma_{l j}\left(\boldsymbol{\Lambda}_{l-}(t)\right)+2 \sigma_{l j}(\overline{\boldsymbol{\rho}}(t))\right] \bar{W}^{l}(t) \sqrt{\Delta t} \\
& +\frac{1}{4} \sum_{l=1}^{n} \sum_{r=1, r \neq l}^{n}\left[\boldsymbol{\sigma}_{l j}\left(\boldsymbol{\Xi}_{r+}(t)\right)+\boldsymbol{\sigma}_{l j}\left(\boldsymbol{\Lambda}_{r-}(t)\right)-2 \boldsymbol{\sigma}_{l j}(\overline{\boldsymbol{\rho}}(t))\right] \bar{W}^{l}(t) \\
& +\frac{1}{4} \sum_{l=1}^{n}\left[\sigma_{l j}\left(\boldsymbol{\Lambda}_{l+}(t)\right)-\boldsymbol{\sigma}_{l j}\left(\boldsymbol{\Lambda}_{l-}(t)\right)\right]\left[\left(\bar{W}^{l}(t)\right)^{2}-1\right] \sqrt{\Delta t} \\
& +\frac{1}{4} \sum_{l=1}^{n} \sum_{r=1, r \neq l}^{n}\left[\sigma_{l j}\left(\boldsymbol{\Xi}_{r+}(t)\right)-\boldsymbol{\sigma}_{l j}\left(\boldsymbol{\Xi}_{r-}(t)\right)\right]\left[\bar{W}^{l}(t) \bar{W}^{r}(t)+V_{r, j}\right] \sqrt{\Delta t}
\end{aligned}
$$

and the supporting values:

$$
\begin{aligned}
& \Upsilon=\overline{\boldsymbol{\rho}}(t)+\boldsymbol{\mu}(\overline{\boldsymbol{\rho}}(t)) \Delta t+\sum_{j=1}^{n} \boldsymbol{\sigma}_{j}(\boldsymbol{\rho}(t)) \Delta W_{j}(t), \\
& \boldsymbol{\Lambda}_{l \pm}=\overline{\boldsymbol{\rho}}(t)+\boldsymbol{\mu}(\overline{\boldsymbol{\rho}}(t)) \Delta t \pm \boldsymbol{\sigma}_{l}(\overline{\boldsymbol{\rho}}(t)) \sqrt{\Delta t} \\
& \mathbf{\Xi}_{l \pm}=\overline{\boldsymbol{\rho}}(t) \pm \boldsymbol{\sigma}_{l}(\overline{\boldsymbol{\rho}}(t)) \sqrt{\Delta t}
\end{aligned}
$$

The random matrix $\mathbf{V}$ is defined as:

$$
V_{r, j}(t)=\left\{\begin{array}{l} 
\pm 1 \quad \text { with } \quad p=\frac{1}{2} \quad \text { if } \quad r<j \\
-1 \quad \text { if } \quad r=j \\
-V_{j, r}(t) \quad \text { if } \quad r>j,
\end{array}\right.
$$

where $p$ indicates the probability. It has to be emphasised that such a scheme does not involve the computation of multiple stochastic integrals, thus its strong order of convergence is expected to be at maximum 1.0.

\subsubsection{Weak and strong order of convergence for temporal integrators}

The order of convergence can be measured in the strong and weak sense, for which the strong and weak errors are respectively defined for a particular time $\tau$ and a group of trajectories $\Gamma=\left\{\gamma_{1}, \gamma_{2}, \ldots, \gamma_{m}\right\}$ as

$$
\boldsymbol{\epsilon}_{s}=\left\langle\left|\overline{\boldsymbol{\rho}}^{\gamma}(\tau)-\overline{\boldsymbol{\rho}}_{\text {exact }}^{\gamma}(\tau)\right|\right\rangle_{\gamma \in \Gamma} \quad \text { and } \quad \boldsymbol{\epsilon}_{w}=\left|\left\langle\overline{\boldsymbol{\rho}}^{\gamma}(\tau)\right\rangle_{\gamma \in \Gamma}-\left\langle\overline{\boldsymbol{\rho}}_{\text {exact }}^{\gamma}(\tau)\right\rangle_{\gamma \in \Gamma}\right|,
$$


where $\overline{\boldsymbol{\rho}}^{\gamma}(\tau)$ refers to the numerical density cell averages at time $\tau$ following trajectory $\gamma, \overline{\boldsymbol{\rho}}_{\text {exact }}^{\gamma}(\tau)$ denotes the exact or reference solution which is considered to be the true solution of the stochastic equation, the ensemble average $\langle\cdot\rangle$ is taken over the trajectories $\gamma \in \Gamma$, and the norm $|\cdot|$ can be evaluated as an $L^{1}$-norm.

In Fig. 2 we evaluate the strong and weak errors for the described stochastic integrators. They are obtained by simulating equation (43) in the simplified case of geometric Brownian motion, for which $\boldsymbol{\mu}(\overline{\boldsymbol{\rho}}(t))=-\overline{\boldsymbol{\rho}}(t)$ and $\sigma(\bar{\rho}(t))=0.5 \bar{\rho}(t)$, thus eliminating the spatial derivatives. As a result, the temporal evolution of the density for a cell $j$, which is independent from the rest of cells, follows

$$
d \bar{\rho}_{j}(t)=-\bar{\rho}_{j} d t+0.5 \bar{\rho}_{j} \overline{\mathcal{W}}_{j} d t
$$

with the cell averaged white noise $\overline{\mathcal{W}}_{j}$ defined as in (34). For the simulation we selected $\bar{\rho}_{j}(0)=1$. Geometric Brownian motion is useful to compute the strong and weak errors since the exact solution in analytically known [66].

The results in Fig. 2 (a) and (b) depict the strong and weak order of convergence for the temporal integrators. Concerning the former, as expected the Euler-Maruyama presents an order of 0.5, while Milstein an order of 1.0. Runge-Kutta is expected to have a strong order of at least 0.5 , and in the plot it approaches a value of 1.0.

With respect to the weak order, the whole families of Euler-Maruyama and Milstein solvers are expected to have an order of 1.0, while the Runge-Kutta an order of 2.0. Such theoretical predictions are respected for all schemes, with the exception of the semi-implicit methods which outperforms, giving an order between 1.0 and 2.0.

On Fig. 2 (c) we plot the cpu time against the total number of cells $n$ for each of the temporal integrators. The Euler-Maruyama accounts for $O(n)$ computations, the Milstein for $O\left(n^{2}\right)$, and the Runge-Kutta for $O\left(n^{3}\right)$. However, for $n<100$ we can observe a lower cpu time for Runge-Kutta, if compared with all the other integrators except for the explicit Euler-Maruyama.

\subsection{Positivity of the density through an adaptive time step}

A natural constraint for physical systems is the positivity of the density field, and the numerical solution is expected to satisfy such a requirement. Numerical schemes with the property of preserving the positivity of the density have been studied in the literature, specially in the context of deterministic conservation law PDEs [62, 67]. The strategy is usually to derive a Courant-Friedrichs-Lewy (CFL) condition which imposes a constraint for the maximum $\Delta t$ so that the density always remains non-negative.

For particular discretizations of the numerical fluxes (20) it is also possible to derive a CFL condition for the SPDE in (6). This CFL condition depends on the Gaussian distributions from the white noise (35), as well as on the density profile. Following the derivation provided in [62] for a deterministic gradient flow equation, we proceed to provide an example of the CFL derivation when the upwind discretizations (28) and (41) for the deterministic and stochastic fluxes, respectively, are employed.

Lemma 3.1. Consider the SPDE (6) with initial data $\rho_{0}(x)>0$, together with the semi-discrete finite-volume scheme (22) with the upwind discretizations for the deterministic (28)-(31) and stochastic (35), (41) fluxes. Assume that the SPDE is temporally discretized with a deterministic Euler forward method. Then, the computed cell averages satisfy $\bar{\rho}_{j} \geq 0, \forall j$, provided that the following two CFL conditions for $\Delta t$ hold:

$$
\frac{1}{2}-\lambda_{1} u_{j+\frac{1}{2}}^{+}-\lambda_{2} \mathcal{G}_{j+\frac{1}{2}}^{+} / \sqrt{\rho_{j}^{E} \beta} \geq 0, \quad \frac{1}{2}-\lambda_{1} u_{j-\frac{1}{2}}^{-}-\lambda_{2} \mathcal{G}_{j-\frac{1}{2}}^{-} / \sqrt{\rho_{j}^{W} \beta} \geq 0
$$

where

$$
\lambda_{1}:=\frac{\Delta t}{\Delta x}, \quad \lambda_{2}:=\sqrt{\frac{\Delta t}{\Delta x}}, \quad \overline{\mathcal{G}}_{j}=\overline{\mathcal{W}}_{j} \sqrt{\Delta x \Delta t}=\mathcal{N}(0,1),
$$

and $\mathcal{G}_{j+1 / 2}^{+}, \mathcal{G}_{j+1 / 2}^{-}$are constructed as in (42), so that

$$
\mathcal{G}_{j+1 / 2}^{+}=\max \left(\mathcal{G}_{j+1 / 2}, 0\right), \quad \mathcal{G}_{j+1 / 2}^{-}=\min \left(\mathcal{G}_{j+1 / 2}, 0\right) .
$$

Proof. Firstly, assume that for a given time $t$ the computed solution for the density is known and positive: $\rho_{j}(t) \geq 0$, $\forall j$. The new cell averages following a forward Euler temporal scheme in the finite-volume formulation (22) satisfy

$$
\bar{\rho}_{j}(t+\Delta t)=\bar{\rho}_{j}(t)-\Delta t\left[\frac{F_{d, j+1 / 2}-F_{d, j-1 / 2}}{\Delta x}+\frac{F_{s, j+1 / 2}-F_{s, j-1 / 2}}{\Delta x}\right] .
$$


(a)

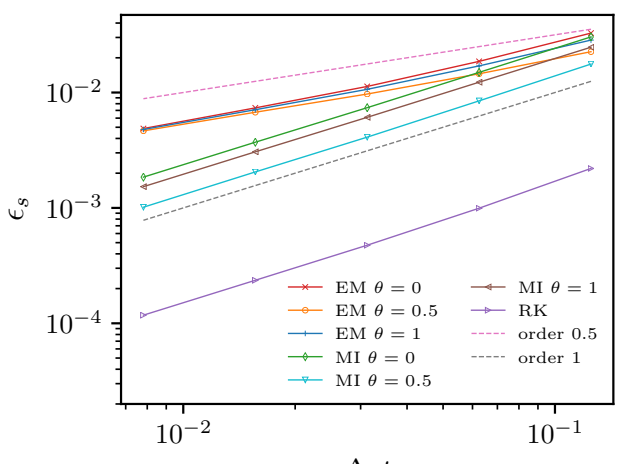

$\Delta t$ (b)

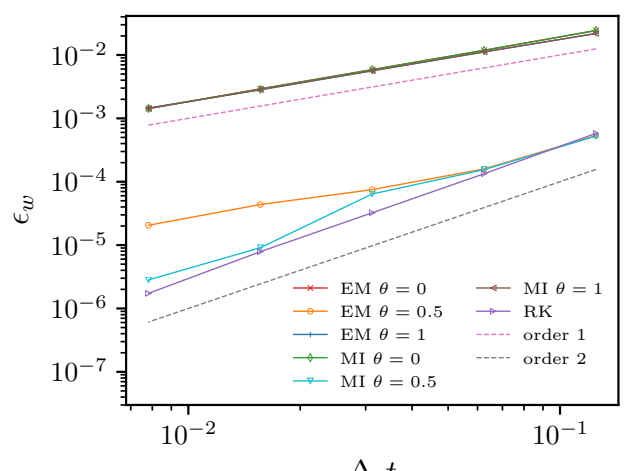

$\Delta t$

(c)

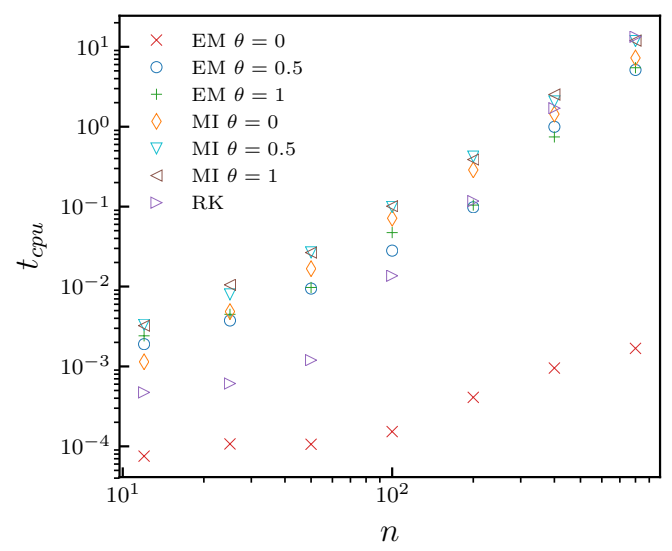

Fig. 2: Strong (a) and weak (b) errors convergence for geometric Brownian motion. In (c) we report the cpu time for each time integration schemes as a function of the number of cells $n$. EM: Euler-Maruyama, MI: Milstein, RK: Runge-Kutta. Explicit $(\theta=0)$, semi-implicit $(\theta=0.5)$ and implicit $(\theta=1)$.

Then, after substituting the deterministic and stochastic fluxes for their upwinded discretizations (28) and (41), respectively, and by employing the notation specified in (58), it follows

$$
\begin{aligned}
\bar{\rho}_{j}(t+\Delta t)= & \frac{1}{2}\left(\rho_{j}^{E}+\rho_{j}^{W}\right)-\lambda_{1}\left[u_{j+\frac{1}{2}}^{+} \rho_{j}^{E}+u_{j+\frac{1}{2}}^{-} \rho_{j+1}^{W}-u_{j-\frac{1}{2}}^{+} \rho_{j-1}^{E}-u_{j-\frac{1}{2}}^{-} \rho_{j}^{W}\right] \\
& -\lambda_{2}\left[\mathcal{G}_{j+\frac{1}{2}}^{+} \sqrt{\rho_{j}^{E} / \beta}+\mathcal{G}_{j+\frac{1}{2}}^{-} \sqrt{\rho_{j+1}^{W} / \beta}-\mathcal{G}_{j-\frac{1}{2}}^{+} \sqrt{\rho_{j-1}^{E} / \beta}-\mathcal{G}_{j-\frac{1}{2}}^{-} \sqrt{\rho_{j}^{W} / \beta}\right] \\
= & \lambda_{1}\left[-u_{j+\frac{1}{2}}^{-} \rho_{j+1}^{W}+u_{j-\frac{1}{2}}^{+} \rho_{j-1}^{E}\right]+\lambda_{2}\left[-\mathcal{G}_{j+\frac{1}{2}}^{-} \sqrt{\rho_{j+1}^{W} / \beta}+\mathcal{G}_{j-\frac{1}{2}}^{+} \sqrt{\rho_{j-1}^{E} / \beta}\right] \\
& +\left[\frac{1}{2}-\lambda_{1} u_{j+\frac{1}{2}}^{+}-\lambda_{2} \mathcal{G}_{j+\frac{1}{2}}^{+} / \sqrt{\rho_{j}^{E} \beta}\right] \rho_{j}^{E}+\left[\frac{1}{2}-\lambda_{1} u_{j-\frac{1}{2}}^{-}-\lambda_{2} \mathcal{G}_{j-\frac{1}{2}}^{-} / \sqrt{\rho_{j}^{W} \beta}\right] \rho_{j}^{W} .
\end{aligned}
$$

Due to the fact that the reconstructed point values for the density $\rho_{j-1}^{E}, \rho_{j+1}^{E}, \rho_{j}^{W}$ and $\rho_{j+1}^{W}$ are non-negative, and bearing in mind that $u_{j-\frac{1}{2}}^{+}, \mathcal{G}_{j-\frac{1}{2}}^{+} \leq 0$ and $u_{j+\frac{1}{2}}^{-}, \mathcal{G}_{j+\frac{1}{2}}^{-} \geq 0$ due to (30) and (59), it follows that $\bar{\rho}_{j}(t+\Delta t) \geq 0, \forall j$, provided that the CFL conditions (57) hold.

The CFL conditions in (57) ensure that the density remains non-negative at all times, no matter the values produced 
by the normal distributions of the white noise spatio-temporal average (35). In the case of a rare event in which the Gaussian distribution produces low-probability values located at the tales of the distribution, $\Delta t$ would be adapted accordingly to ensure the positivity. This adaptive time strategy entails however two main disadvantages. First, it requires to solve at each time step a second-order equation (in one-dimension) or a two-parameter equation in multidimensional problems. Second, since the time-step size is dependent on the random number at each step, higher (or lower) $\Delta t$ may be favored by some random numbers, thus not guaranteeing that the correct Brownian path is followed[68].

Previous works in the literature have already addressed the issue of positivity by means of varied approaches. In the context of FH, the authors of Ref. [35] have effectively opted for introducing cutting functions based on smoothed Heaviside functions which prevent the density from becoming negative. The main drawbacks of this strategy are 1) that, despite reducing the chances of having negative density values, positive densities are not guaranteed, and 2) that it affects the density distribution.

A further alternative to preserve positivity lays in the concept of Brownian trees, which were firstly introduced in [68] in order to address the numerical resolution of stochastic differential equations with variable time steps. The key idea here is that, unlike with deterministic differential equations, it is vital to respect the Brownian path that is formed after evaluating the normal distributions (35). This means that upon advancing our simulation from time $t$ a certain $\Delta t_{1}$ and realising that the density in one of the nodes $j$ has become negative we cannot just simply repeat the time step with a shorter $\Delta t_{2}<\Delta t_{1}$ in order to maintain positivity. The values of the normal distributions after the first trial of advancing $\Delta t_{1}$ have to be respected if the Brownian path is to be preserved. In addition, those values of the normal distributions at $t+\Delta t_{1}$ have to be employed when computing the values at $t+\Delta t_{2}$, even if the jump from $t$ to $t+\Delta t_{1}$ has produced negative densities.

The solution to effectively take the statistical information at $t+\Delta t$ into account when repeating the time step is the so-called Brownian bridge $[69,65]$. It allows the computation of $\overline{\mathcal{W}}_{j}$ in Eq. (34) at an intermediate time step $t+\Delta t / 2$ by means of the formula

$$
\overline{\mathcal{W}}_{j}\left(t+\frac{\Delta t}{2}\right)-\overline{\mathcal{W}}_{j}(t)=\frac{\overline{\mathcal{W}}_{j}(t+\Delta t)-\overline{\mathcal{W}}_{j}(t)}{2}+\mathcal{N}\left(0, \frac{\Delta t}{4}\right) .
$$

As a result, our tactic consists of initially selecting an adequately small $\Delta t$. Then, if after some time the density becomes negative, $\Delta t$ is divided by 2 to compute the intermediate time step from the Brownian bridge (62). If that intermediate state leads to further negative densities, the Brownian bridge is applied as many times as needed. The information at $t+\Delta t$ is saved to be employed once all the intermediate time steps with non-negative densities are computed. A pseudocode to implement the Brownian bridge is written in Algorithm 1. As a remark, the adequate choice of a small initial $\Delta t$ for the simulation is essential to reduce the number of Brownian bridges to a minimum. A compromise is of course needed, since an extremely small $\Delta t$ does not lead to negative densities but requires a high computational cost for the simulation.

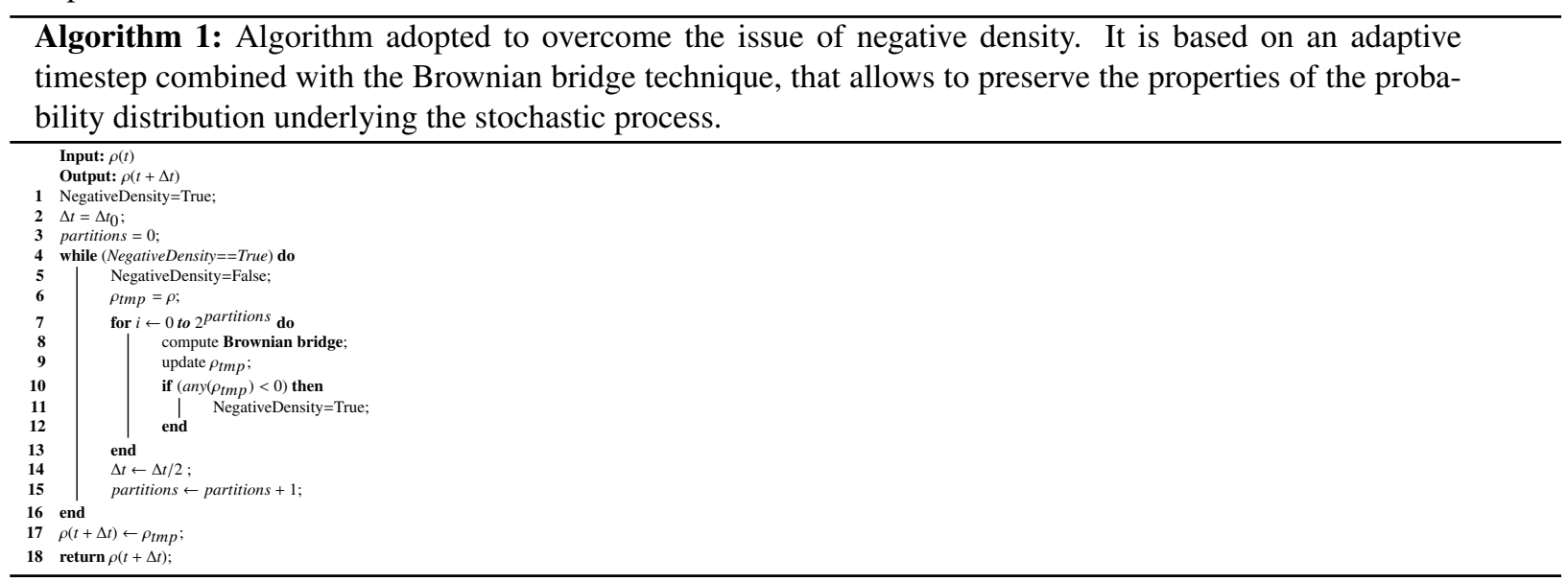




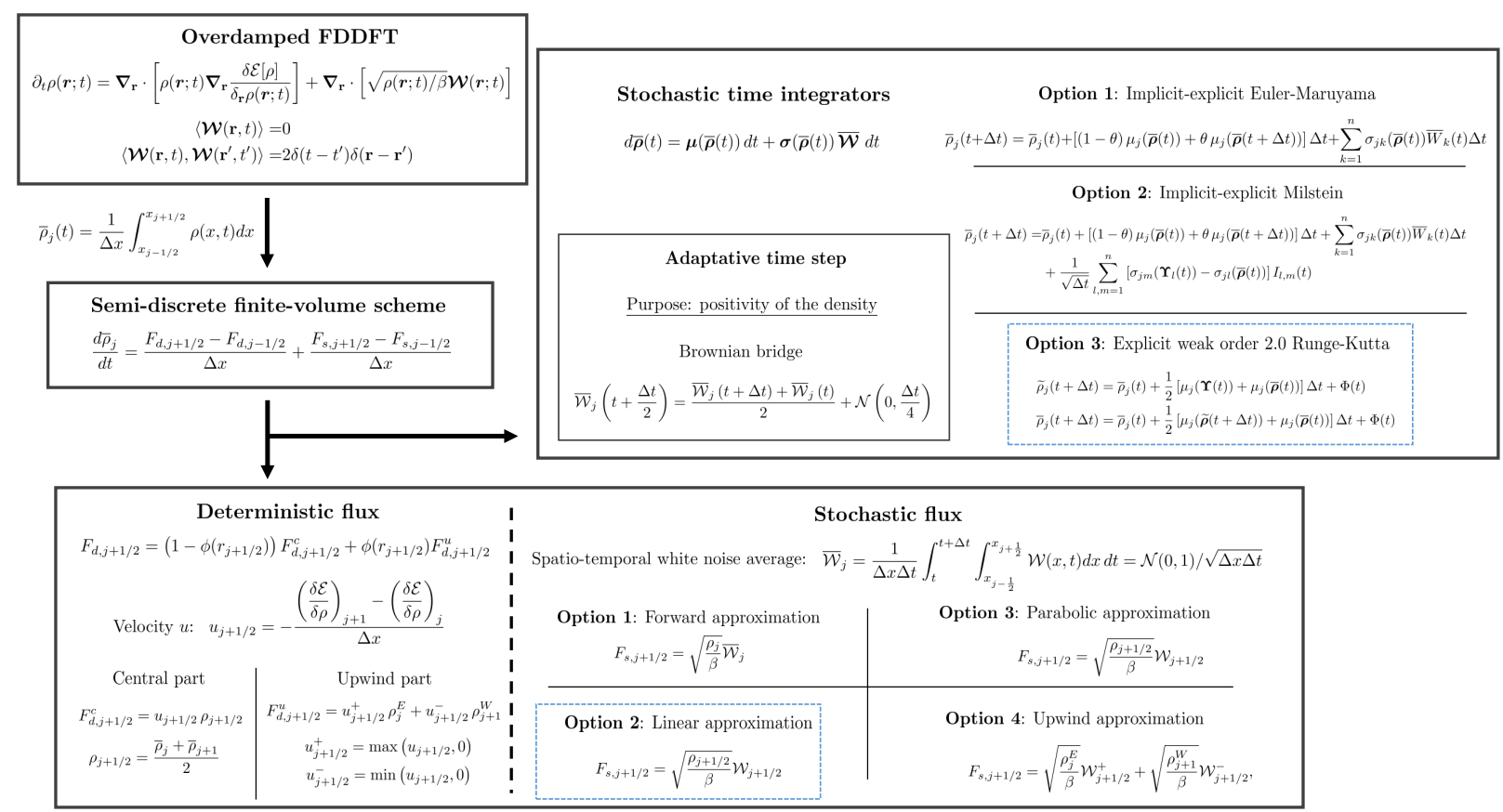

Fig. 3: Flowchart reporting the main equations adopted to solve the overdamped FDDFT with a finite-volume approach. Arrows denote the connections among the different steps. Blue boxes denote the options shown to provide the best accuracy and efficiency to simulate our SPDE (19), as explained in Sect. 4.

\subsection{Boundary conditions}

In this section we analyse the implementation of boundary conditions for the cases of periodic, confined and open systems. For systems with a periodic boundary, it is sufficient to impose

$$
\rho_{0}=\rho_{N} .
$$

For no-flux conditions the boundary conditions to impose in the fluxes are

$$
F_{j \pm 1 / 2}=0 \text { for } j=0, N .
$$

Open systems in thermal and chemical equilibrium with a reservoir can be represented by a $\mu V T$ ensemble with constant grand potential $\Omega[\rho]=\mathcal{E}[\rho]-\mu \int \rho d x$, where $\mathcal{E}[\rho]=\mathcal{F}[\rho]+\int V(x) \rho d x$ with $\mathcal{F}[\rho]$ being the Helmholtz free-energy functional, $V(x)$ the external potential acting on the system and $\mu$ the chemical potential. Using the fact that the functional derivative of $\Omega$ with respect to $\rho$ is null in equilibrium, we obtain $\delta \mathcal{E}[\rho] / \delta \rho=\mu$. Since the system is assumed to be in contact with a reservoir at temperature $T_{\text {res }}$ and chemical potential $\mu_{\text {res }}$, the corresponding boundary condition to be applied to compute the velocities $u_{j+1 / 2}$ at the boundaries in (29) is

$$
\left(\frac{\delta \mathcal{E}}{\delta \rho}\right)_{0}=\left(\frac{\delta \mathcal{E}}{\delta \rho}\right)_{N}=\mu_{\mathrm{res}},
$$

with $\mu_{\text {res }}$ being the chemical potential of the reservoir. From the value of $\mu_{\text {res }}$ one can compute the density by solving (10) for a fixed value of $\delta \mathcal{E} / \delta \rho$. This implies that the values of $\rho_{0}, \rho_{N}$ and any additional ghost node are imposed from (65) for all times. Depending on the particular choice of free energy in (7), it may be possible to converge to different density profiles depending on the initial condition for the iterative algorithm to solve (10). This open boundary condition imposes a positive or negative flux of mass through the boundary, and as a result the total mass is not conserved in time. 


\section{Numerical applications}

In this section we provide tests of the numerical schemes developed in Sect. 3. Initially, in subsection 4.1 we conduct a simulation with a purely-diffusive ideal-gas free energy with noise and without external fields or interparticles potentials. There are several theoretical results for such systems [38, 35] allowing us to benchmark the statistical correlation and the structure factor from our numerical schemes. Further validation of the schemes will be offered via comparison with our own MD simulations. The results of these tests show that the Runge-Kutta temporal integrator (49) and the linear approximation of the stochastic flux (37)-(38) accomplish the best accuracy and efficiency to simulate our SPDE (19). This choice is maintained in examples that follow.

Secondly, we provide a simulation for an ideal gas with a local confining external potential $V(x)$, in order to test the mean and variance of the density, the spatial correlation and the decay of the discrete free energy in time.

The simulations of ideal gases are also compared with results from MD simulations using the software LAMMPS [70].

\subsection{Ideal-gas system in equilibrium}

Consider the SPDE in (6) without any external or interaction potential $(V(x)=W(x)=0)$ and applied to the classical ideal-gas free energy

$$
\mathcal{E}[\rho]=\beta^{-1} \int \rho(\ln (\rho)-1) d x
$$

leading to a diffusive equation with multiplicative noise of the form

$$
\partial_{t} \rho=\Delta \rho / \beta+\nabla \cdot[\sqrt{\rho / \beta} \mathcal{W}(x, \rho)] .
$$

The initial density profile is taken as the equilibrium one, with a constant value in all cells of $\rho_{j}=0.5$ and a total number of particles of $N=1,000$ for the MD simulation. The mean density profile $\bar{\rho}$ at any time, taken as the mean of the density ensemble averages at every cell, is expected to remain as $\bar{\rho} \approx 0.5$ throughout the simulation due to the equilibrium state. The number of cells in the domain is $n=40$, the cell size is $\Delta x=50$ and the time step is computed as $\Delta t=0.1 \Delta x^{2}$ (selected as in [35]), the number of trajectories is 100 , and the number of time steps is 2,000 , unless otherwise stated in each of the tests. The boundary conditions are periodic and the parameter $\beta$ is fixed at $\beta=1$.

The objective is to evaluate how the different space and temporal numerical methods perform by focusing on four different statistical properties at equilibrium: variance, spatial correlation, time correlation and structural factor. These tests are usually employed in the literature for the validation of stochastic numerical schemes for FH [38, 35, 71]. The advantage of testing these properties at equilibrium is that their exact theoretical values are known and can be used for comparison purposes. Density fluctuations of an system with fixed volume $V$ can be computed as $\left\langle\delta \rho^{2}\right\rangle=\rho^{2}\left\langle\delta N^{2}\right\rangle / N^{2}$, where $N$ and $\left\langle\delta N^{2}\right\rangle$ denote average and variance of the number of particles in $V$, respectively. As shown in Ref. [2], the variance is given by:

$$
\left\langle\delta N^{2}\right\rangle=-T \frac{\bar{N}^{2}}{V^{2}}\left(\frac{\partial V}{\partial p}\right)_{T}
$$

where $T$ and $p$ are the temperature and pressure of the system, respectively. Employing the equation of state (in reduced units) for an ideal gas, $p V=N T$, we obtain $\left\langle\delta N^{2}\right\rangle=N$. In the case of infinite systems, the fluctuations of an ideal gas are spatially uncorrelated, namely $\left\langle\delta \rho_{i}(t) \delta \rho_{j}(t)\right\rangle=\left\langle\delta \rho^{2}\right\rangle \delta_{i j}^{K}$. However, for finite systems the constraint on conservation of mass introduces correlations [38]. Expressing the space correlations of density fluctuations as $\left\langle\delta \rho_{i}(t) \delta \rho_{j}(t)\right\rangle=A \delta_{i j}^{K}+B$, then conservation of mass dictates $\sum_{i}\left\langle\delta \rho_{i}(t) \delta \rho_{j}(t)\right\rangle=0$, which corresponds to the constraint $B=-A / n$, with $n$ being the total number of cells. Moreover, in the limit $n \mapsto \infty$ the fluctuations for an infinite system have to be recovered, thus $A=\left\langle\delta \rho^{2}\right\rangle$. It follows that the spacial correlation for the closed system can be expressed as:

$$
\left\langle\delta \rho_{i}(t) \delta \rho_{j}(t)\right\rangle=\left\langle\delta \rho^{2}\right\rangle\left(\delta_{i j}^{K}-\frac{1}{n}\right) .
$$

The expression for the variance allows us also to obtain a quick estimation of the minimum cell size for which, due to thermal fluctuations, negative density values are likely to occur. The expected value of the density fluctuations for an ideal gas can be expressed as its standard deviation $\sqrt{\left\langle\delta \rho^{2}\right\rangle}=\sqrt{\rho / \Delta V}$. Thus, with a confidence of 99.7\%, 
the maximum values of the density fluctuations will be $\left.\sqrt{\left\langle\delta \rho^{2}\right\rangle}\right|_{\max } \sim 3 \sqrt{\frac{\rho}{\Delta V}}$. It follows that the noise fluctuations give unphysical density values lower than zero with a probability higher than $0.3 \%$ when the following condition is verified:

$$
3 \sqrt{\frac{\rho}{\Delta V}} \gtrsim \rho \quad \text { or, equivalently } \quad \Delta V \lesssim \frac{3}{\sqrt{\rho}}
$$

Eventually, in subsubsection 4.1.5 we provide a discussion to elucidate which temporal integrator and spatial discretization of the stochastic test performs more accurately and efficiently, bearing in mind the computational cost and based on the results from the four tests. The justified choices, which are the Runge-Kutta temporal integrator (49) and linear approximation of the stochastic flux (37)-(38), are employed during the four tests, in the sense that the Runge-Kutta temporal integrator is employed when evaluating the different spatial discretizations, and the linear approximation of the stochastic flux is employed when evaluating the temporal integrators.

\subsubsection{Standard deviation}

For this test we aim to evaluate how the standard deviation of the density varies depending on the number of particles per cell $N_{c}$. We keep the total length and the total number of particles in the domain as constant, and we only vary the number of particles per cell by enlarging or shortening the cell size $\Delta x$. Consequently this analysis helps to elucidate how changing the finite-volume lattice size affects the numerical statistical properties. The mean density of the profile is $\bar{\rho}=N /(n \Delta x)$.

As shown above, the theoretical standard deviation of the diffusion SPDE (67) applied in finite systems in equilibrium satisfies

$$
{\sqrt{\left\langle\delta \rho^{2}\right\rangle_{\text {theory }}}}=\frac{\langle\rho\rangle}{\sqrt{N_{c}}} \sqrt{1-\frac{1}{n}} .
$$

As a remark, in spite of the fact that $\sqrt{\left\langle\delta \rho^{2}\right\rangle}$ theory holds for all $N_{c}$, previous studies [36, 71] have pointed out that there should be a minimum of 5-10 particles per cell to recover the microscopic statistical properties by means of FH. This occurs because with such low number of particles per cell the particle fluctuations are not accurately modelled with the multiplicative noise in (67).

The results of this study are displayed in Fig. 4, depicting a comparison of the temporal schemes (a) and spatial discretizations (b) against the theoretical standard deviation (71) and the one computed from MD. From both plots we can observe how all the schemes approximate correctly the standard deviation for $N_{c}>5$. Below that number of particles per cell the standard deviations deviate from the expected ones. This result chimes in with the minimum number of 5-10 particles per cell necessary to recover the statistical properties in FH.

There are no remarkable differences between the temporal integrators or spatial discretizations for the stochastic flux.

\subsubsection{Time correlations}

The objective of this test is to measure the time correlation of the density in one specific cell of the domain. The normalized time correlation function is defined as

$$
C_{T}(t)=\frac{\left\langle\delta \rho_{i}(t) \delta \rho_{i}(0)\right\rangle}{\left\langle\delta \rho_{i}(0) \delta \rho_{i}(0)\right\rangle},
$$

where $\delta \bar{\rho}_{i}(t)=\bar{\rho}_{i}(t)-\bar{\rho}$. The time correlation function expected to decay in time for any process in equilibrium, including the diffusion equation (67). Previous studies [38] have compared the numerical results with the Fourier transform of the time correlation (72), which is denoted as the spectral density and for which there are explicit expressions available. In spite of this, these exact expressions for the spectral density do not take into account the finite-size effects from the numerical simulations, leading to a lack of agreement in the results [38].

We have then decided to compare the results obtained from the numerical schemes in Sect. 3 with MD simulations only, which indeed take into account the finite-size effects of the numerical simulation. The results are displayed in Fig. 5. For all schemes we evidence a clear decay in time of the time correlation. Concerning the temporal integrators, the explicit ones $(\theta=0)$ tend to be closer to the MD simulations for the initial short times, while the implicit ones $(\theta=1)$ provide a better approximation in the long-time regimes. With respect to the spatial discretizations for the stochastic flux, the upwind one deviates the most from MD, while the rest of them behave similarly. 
(a)

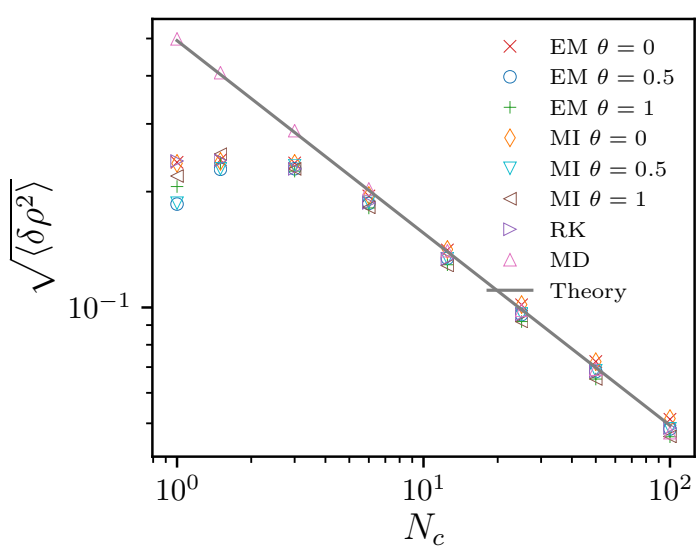

(b)

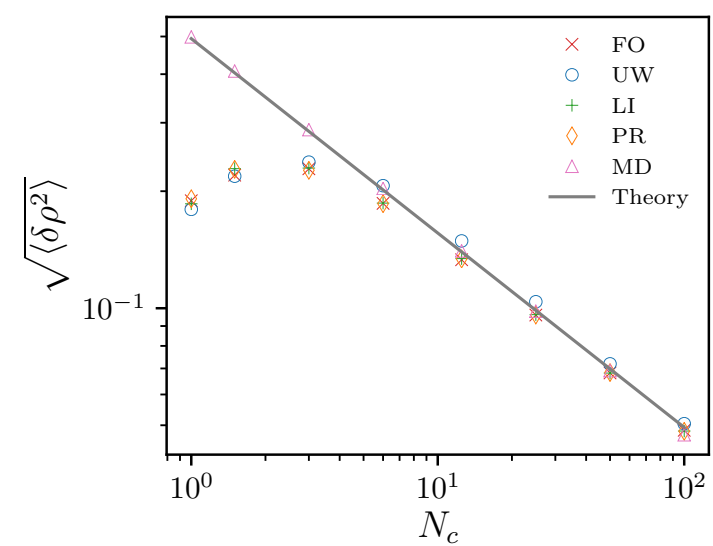

Fig. 4: Standard deviation $\sqrt{\left\langle\delta \rho^{2}\right\rangle}$ as a function of the number of particles per cell $N_{c}$ (or, equivalently, as a function of the cell size given that total volume and density of the system are kept constant throughout the simulations), for an ideal gas in equilibrium. (a) Temporal integrators. EM: Euler-Maruyama, MI: Milstein, RK: Runge-Kutta, MD: Molecular dynamics. Explicit $(\theta=0)$, semi-implicit $(\theta=0.5)$ and implicit $(\theta=1)$. (b) Spatial discretizations of the stochastic flux. FO: Forward (36), UW: Upwind (41)-(42), LI: Linear (37)-(38), PR: Parabolic (39)-(40), MD: Molecular dynamics, Theory: Eq. 71.

(a)

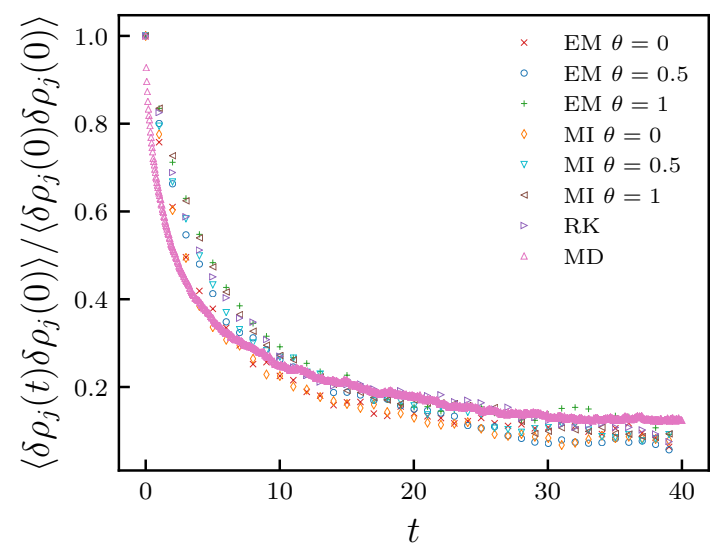

(b)

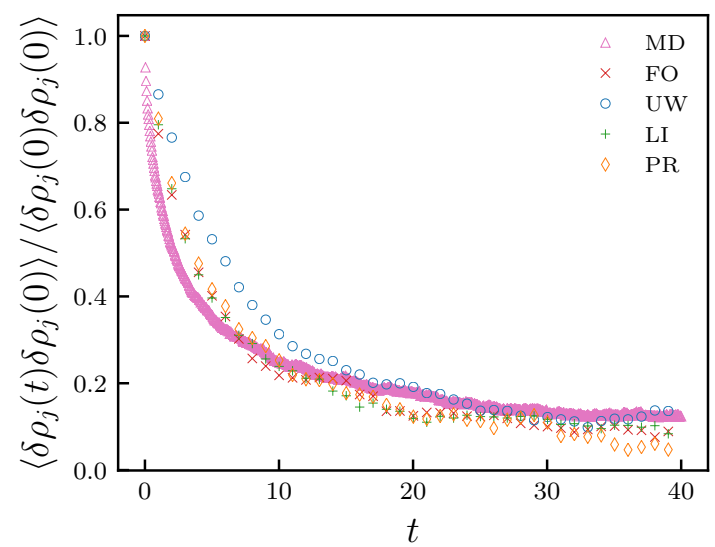

Fig. 5: Temporal decay of the normalized time correlation $C_{T}$, defined as in (72), for an ideal-gas system in equilibrium (a) Temporal integrators. EM: Euler-Maruyama, MI: Milstein, RK: Runge-Kutta, MD: Molecular dynamics. Explicit $(\theta=0)$, semi-implicit $(\theta=0.5)$ and implicit $(\theta=1)$. (b) Spatial discretizations of the stochastic flux. FO: Forward (36), UW: Upwind (41)-(42), LI: Linear (37)-(38), PR: Parabolic (39)-(40), MD: Molecular dynamics.

\subsubsection{Spatial correlations}

This test seeks to evaluate whether the proposed numerical schemes in Sect. 3 satisfy the exact spatial correlation for finite-size systems derived above:

$$
\left\langle\delta \rho_{i}(t) \delta \rho_{j}(t)\right\rangle=\frac{\langle\rho\rangle}{\Delta x}\left(\delta_{i j}-\frac{1}{n}\right) .
$$

Contrary to the infinite-domain case where there are no spatial correlations between adjacent cells, for the finite-size case there is an extra term $1 / n$ which decreases as the number of cells $n$ increases.

The results of this test are depicted in Fig. 6, with the normalized spatial correlation

$$
C_{S}(t)=\frac{\left\langle\delta \rho_{i}(t) \delta \rho_{j}(t)\right\rangle}{\left\langle\delta \rho_{i}(0) \delta \rho_{i}(0)\right\rangle}
$$


with $\delta \bar{\rho}_{i}(t)=\bar{\rho}_{i}(t)-\bar{\rho}$, plotted for each of the numerical schemes, the MD simulations and the exact expression (73). The main conclusion is that most of the temporal integrators and spatial discretizations approximate adequately the theoretical expression (73), as it is depicted in Fig. 6. The fully explicit and implicit Euler-Maruyama and Milstein slightly deviate with respect to the theoretical spatial correlation in the cells adjacent to the central cell, while the semi-implicit schemes perform correctly.

(a)

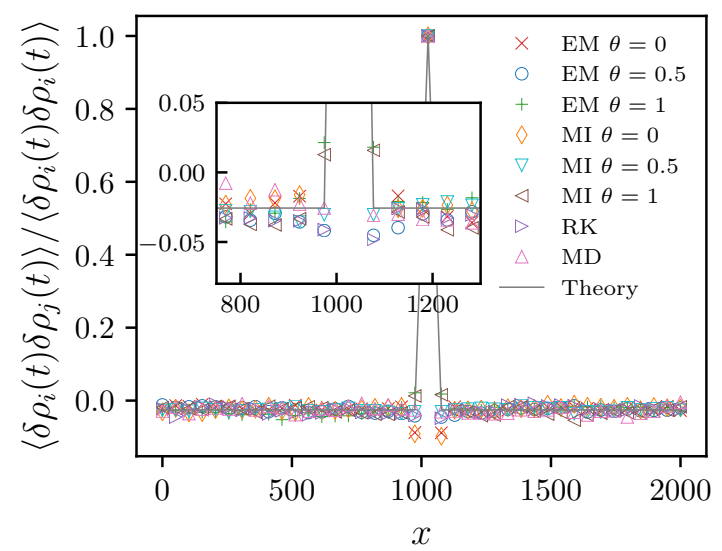

(b)

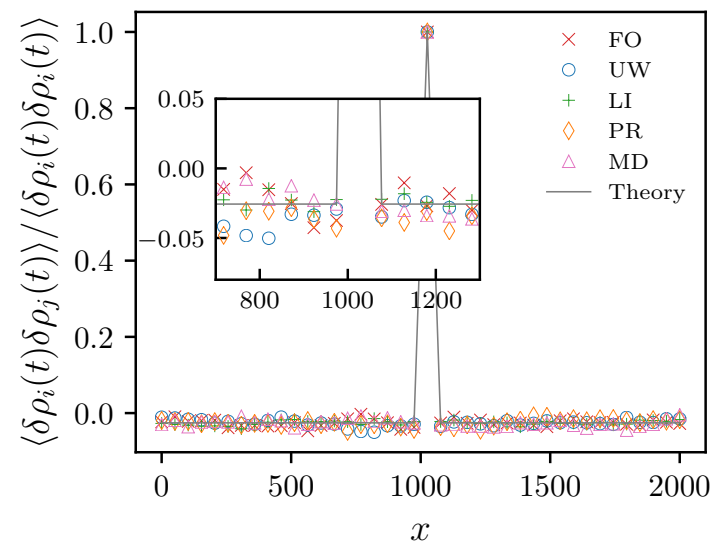

Fig. 6: Normalized spatial correlation (74) for an ideal-gas system in equilibrium. (a) Temporal integrators. EM: Euler-Maruyama, MI: Milstein, RK: Runge-Kutta, MD: Molecular dynamics. Explicit $(\theta=0)$, semi-implicit $(\theta=0.5)$ and implicit $(\theta=1)$. (b) Spatial discretizations of the stochastic flux. FO: Forward (36), UW: Upwind (41)-(42), LI: Linear (37)-(38), PR: Parabolic (39)-(40), MD: Molecular dynamics.

\subsubsection{Structure factor}

This test evaluates how the structure factor $S$ at equilibrium is approximated by the temporal and spatial discretizations. Even though its general form satisfies (17), its theoretical expression for an ideal gas without external potential is given by (18), so that for the current numerical simulation with $\beta=1$ it follows that $S /\langle\rho\rangle=1$.

The discrete structure factor is computed from Eqs (11)-(12). Firstly, the discrete spatial Fourier transform of the density satisfies:

$$
\hat{\rho}(\lambda)=\frac{1}{n} \sum_{j} \bar{\rho}_{j} e^{-i \lambda x_{j}} .
$$

Subsequently, the structure factor follows from

$$
S(\lambda)=\frac{\left\langle\delta \hat{\rho}(\lambda) \delta \hat{\rho}^{*}(\lambda)\right\rangle}{n \Delta x},
$$

where $\delta \hat{\rho}(\lambda)=\hat{\rho}(\lambda)-\langle\hat{\rho}(\lambda)\rangle$ and $\hat{\rho}^{*}$ denotes the complex conjugate of $\hat{\rho}$.

The results of this test for the structure factor at equilibrium are depicted in Fig. 7. The theoretical value of the structure factor, along with the performed MD simulations, allows us to judge whether the temporal integrators and spatial discretizations perform accurately. On the one hand, from Fig. 7(a) one can appreciate how the explicit Euler-Maruyama and Milstein temporal integrators overestimate the structure factor for large $\lambda$, while their implicit versions underestimate it for large $\lambda$ too. The semi-implicit schemes and the Runge-Kutta behave correctly, and the small damping in the numerical structure factor for all $\lambda$ is due to the choice of the hybrid deterministic flux, as it was explained from Fig. 1. On the other hand, from the spatial discretizations of the stochastic flux there is a clear deviation when applying the upwind form. In addition, the forward discretization seems to slihgtly oscillate for lower $\lambda$. The rest of discretizations approximate the theoretical value correctly, with the small damping already mentioned.

\subsubsection{Temporal integrators and spatial discretization of the stochastic flux}

With respect to the temporal integrators, both the fully explicit and implicit Euler-Maruyama and Milstein present certain deviations in the time correlation (Fig. 5), spatial correlation (Fig. 6) and structure factor (Fig. 7). Their semiimplicit versions and the Runge-Kutta behave similarly in all tests, and approximate adequately the theoretical and 
(a)

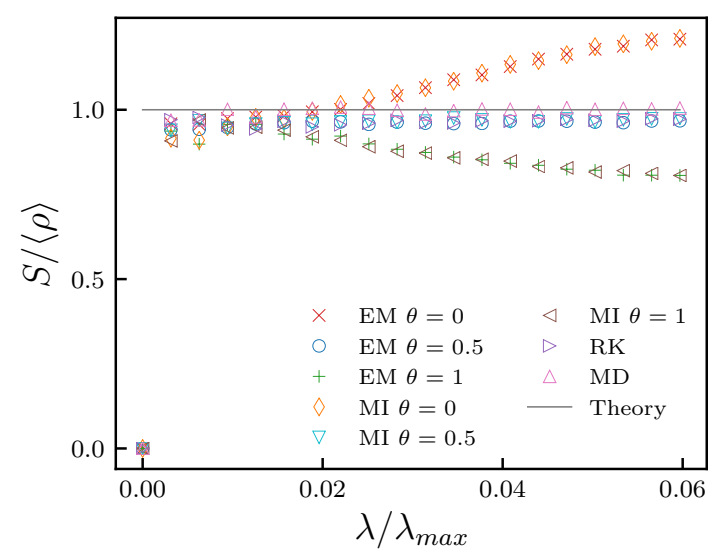

(b)

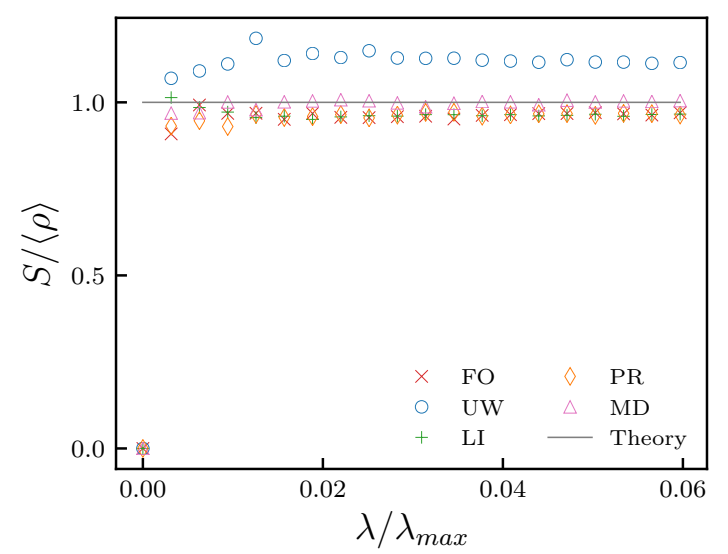

Fig. 7: Structure factor (76) for an ideal-gas system in equilibrium. (a) Temporal integrators. EM: Euler-Maruyama, MI: Milstein, RK: RungeKutta, MD: Molecular dynamics. Explicit $(\theta=0)$, semi-implicit $(\theta=0.5)$ and implicit $(\theta=1)$. (b) Spatial discretizations of the stochastic flux. FO: Forward (36), UW: Upwind (41)-(42), LI: Linear (37)-(38), PR: Parabolic (39)-(40), MD: Molecular dynamics.

MD results. Their relative costs are compared by means of Fig. 2. While the cost of Runge-Kutta escalates with order $O\left(n^{3}\right)$, the cost of the semi-implicit Euler-Maruyama and Milstein has an order of $O\left(n^{2}\right)$. However, due to the different constant coefficient in the cost, the plot clearly shows that for $n<100$ the Runge-Kutta cost is lower than the semi-implicit schemes, while for $n>100$ it is higher.

The Milstein scheme, which guarantees a higher strong order convergence, was tested because in previous works it performed well in conjunction with adaptive time-step algorithms based on Brownian trees [65]. However, from the simulation results, we observed that the higher computational cost of this numerical method did not lead to a enhanced accuracy compared to the implicit Euler-Maruyama and to the weak Runge-Kutta schemes. Because of these reasons and together with the fact that in the simulations of this work $n<100$, we select the Runge-Kutta temporal integrator.

Concerning the spatial discretization of the stochastic flux, the upwind choice does not approximate well the time correlation and structure factor, while the forward approximation presents some deviation in the structure factor for short $\lambda$. Hence the best choices are the linear and parabolic approximations, which behave similarly in all test cases. We select the linear approximation due to its lower cost since it only depends on the density and white noise cell averages of two cells and not four.

\subsection{Ideal-gas system out of equilibrium}

For this example we consider a free energy which includes the effects of a double-well external potential, so that

$$
\mathcal{E}[\rho]=\int \rho / \beta(\ln (\rho)-1) d x+\int V(x) \rho d x,
$$

and the shape of the external potential satisfies

$$
V(x)=5\left[\left(\frac{x}{n \Delta x / 2}\right)^{4}-\left(\frac{x}{n \Delta x / 2}\right)^{2}\right] .
$$

Numerical simulations for deterministic gradient flow equations with the free energies of the form (77)-(78) have already been provided in [62]. Here the objective is to evaluate how the numerical scheme in Sect. 3 for the FDDFT (19) with the free energy (77)-(78) performs by comparing with MD simulations. We also include a comparison with the corresponding deterministic DDFT, which is obtained in the mean-field limit (the most-likely path of FDDFT as noted in the Introduction).

The simulation is performed in a mesh where the number of cells is $n=40$, each of them with width $\Delta x=5$. The time steps are $\Delta t=1$ and the number of time steps is $n_{t}=2000$. The ensemble averages are computed from a number 
of trajectories of $n_{t r a j}=1000$. We select $\beta=1$. The MD simulation is performed by simulating $N=200$ particles, while the deterministic DDFT simulation applies the numerical scheme in [62] for gradient flow equations.

The results are depicted in Fig. 8. Fig. 8 (a) displays the ensemble average of the density profile at different times. The three simulations provide similar results and we can conclude that the three approaches are comparable when evaluating the ensemble average profile. Concerning the standard deviation results in Fig. 8 (b), we find that FDDFT matches with MD and the theoretical results in (71), while DDFT, being deterministic, presents zero standard deviation. As already mentioned, the FDDFT values of the standard deviation are slightly lower than the MD and theoretical ones due to the choice of the deterministic flux in a similar fashion to Figs1-4. Fig. 8 (c) shows the spatial correlation computed as in Eqs (73)-(74), with the MD and FDDFT results approximating correctly the finite-size theoretical expression in (73). DDTF does not have any spatial correlation due to the lack of fluctuations. Finally, in 8 (d) the temporal evolution of the free-energy functional depending on the ensemble average density is plotted. For the DDFT case one can appreciate that there is decay at all times, while for MD and FDDFT there are short increases of the free energy triggered by the fluctuations, in spite of the fact that during the evolution there is a general decay in the free energy.

(a)

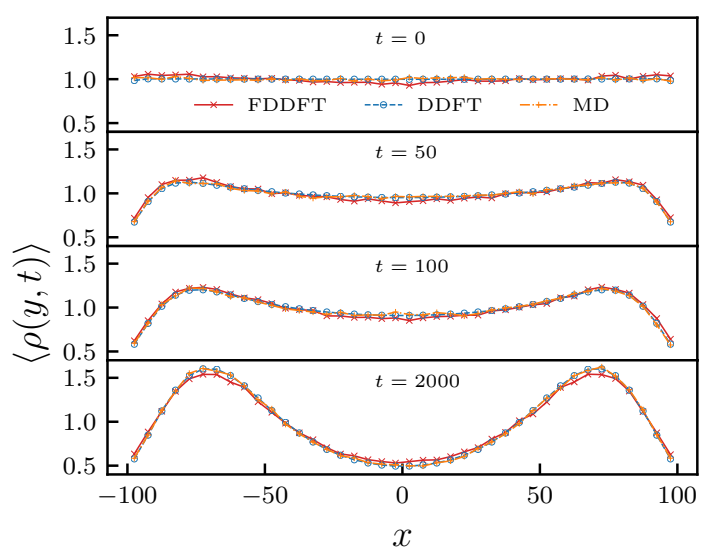

(c)

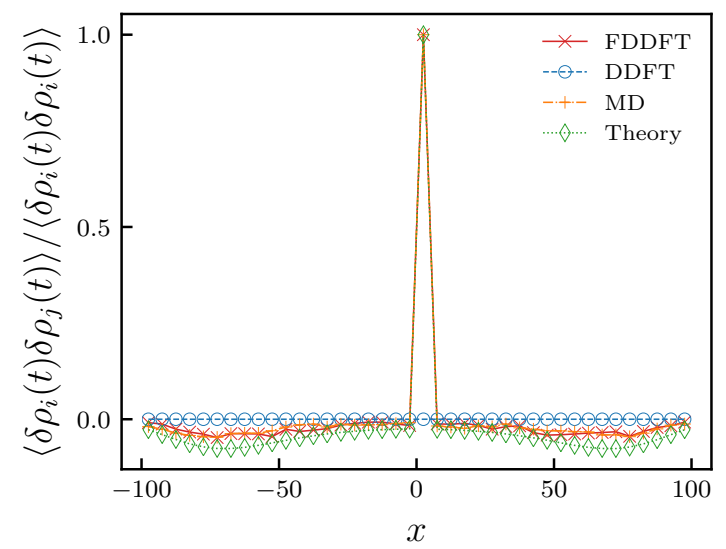

(b)

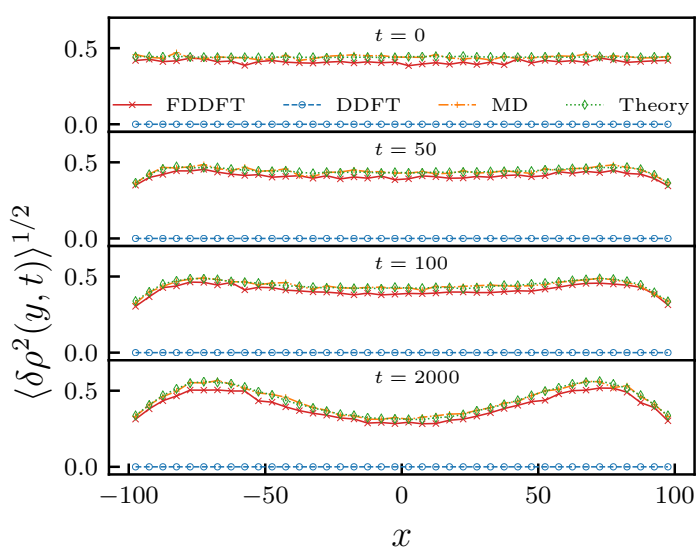

(d)

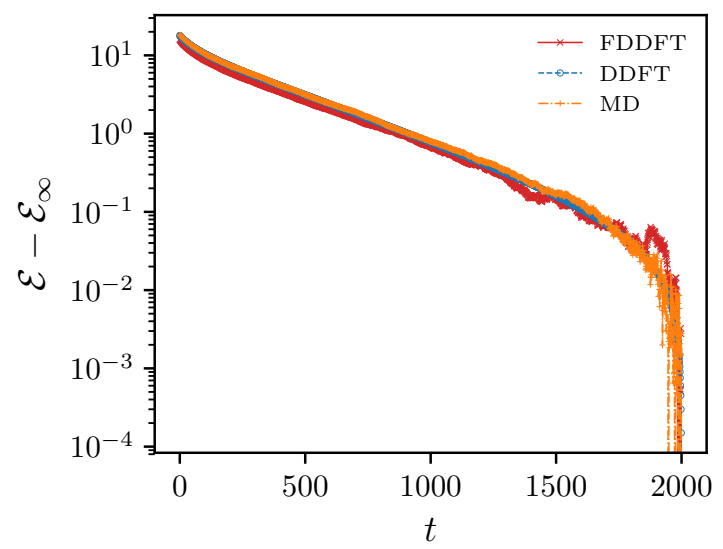

Fig. 8: Time evolution of mean density (a) and density standard deviation (b) fields computed with FDDFT, DDFT and MD simulations. A comparison in terms of steady state spatial correlations is reported in (c). In (d), we report the decrease in time of the energy functional of the mean density. 
(a)

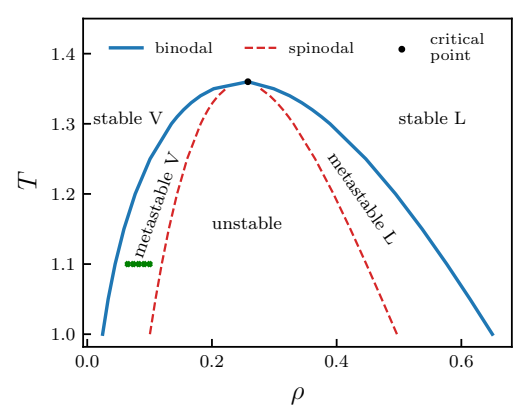

(b)

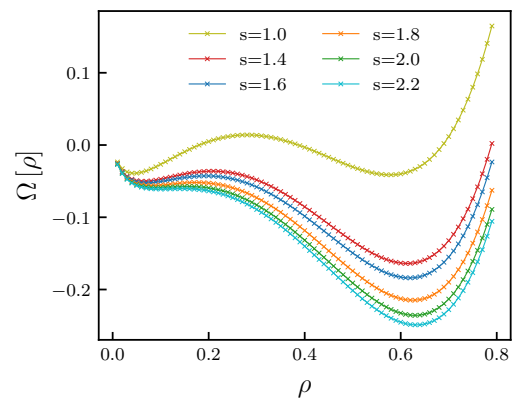

(c)

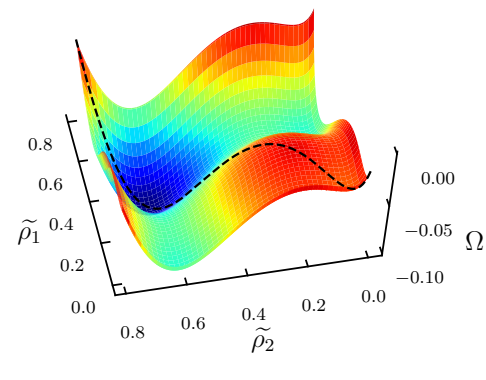

Fig. 9: On panel (a), we report the bulk phase diagram for the discretized LJ system. Panel (b) shows the grand free-energy landscape as function of the system density for some supersaturation ratios adopted in this study. In (c) we provide an example of free-energy landscape for systems with a non-uniform density field, with two varying densities $\widetilde{\rho}_{1}$ and $\widetilde{\rho}_{2}$. The dotted black line denotes the curve corresponding to bulk uniform systems.

\subsection{Homogeneous nucleation in Lennard-Jones systems}

The importance of fluctuations during phase transitions is crucial when considering the homogeneous vapourliquid transition of a Lennard-Jones fluid. Within the framework of DFT, the fluid density profiles of a one-dimensional open system that can exchange particles with a reservoir at constant temperature and chemical potential $\mu$, can be obtained from an unconstrained numerical minimization of the grand free-energy functional

$$
\Omega[\rho(x)]=\mathcal{F}[\rho(x)]+\int(V(x)-\mu) \rho(x) d x .
$$

In general, $\mathcal{F}[\rho(x)]$ is not analytically obtainable from first principles, except in few cases, i.e. ideal gases and hard-sphere fluids. In the remaining cases, $\mathcal{F}[\rho(x)]$ is either numerically obtained from atomistic simulations or is approximated by means of perturbation expansions around a known free energy [19]. Similarly to previous works on DFT [25, 29], we approximate $\mathcal{F}[\rho(x)]$ of an $\mathrm{LJ}$ fluid according to the first-order Barker-Henderson perturbation theory expansion around the hard-sphere fluid free energy [72], namely as

$$
\mathcal{F}[\rho(x)]=\int\left\{f_{\mathrm{ID}}[\rho(x)]+\rho(x) f_{\mathrm{HS}}(\rho(x))\right\} d x+\frac{1}{2} \iint \rho(x) \rho\left(x^{\prime}\right) W\left(x, x^{\prime}\right) d x d x^{\prime},
$$

where $f_{\mathrm{ID}}, f_{\mathrm{HS}}$ and $W\left(x, x^{\prime}\right)$ denote ideal gas, hard-sphere repulsive interactions and $\mathrm{LJ}$ attractive contributions, respectively. The free energy of an ideal gas is given by

$$
f_{\mathrm{ID}}[\rho(x)]=k_{\mathrm{B}} T \rho\left(\ln \left(\lambda^{3} \rho\right)-1\right),
$$

where $\lambda$ is the thermal de Broglie wavelength. The hard sphere free-energy density $f_{\mathrm{HS}}$ is obtained from the CarnahanStarling equation of state for the hard sphere fluid, which reads [73]

$$
f_{H S}(\rho(x))=k_{\mathrm{B}} T\left(\frac{4 \eta-3 \eta^{2}}{(1-\eta)^{2}}\right), \quad \text { with } \quad \eta=\frac{\pi}{6} \rho \sigma^{3}
$$

and with $\sigma$ being the hard sphere diameter and set to one in this work. Finally the LJ (attractive) contributions are taken into account by the following expression:

$$
W\left(x, x^{\prime}\right)= \begin{cases}-1.2 \pi \epsilon & \text { if }\left|x-x^{\prime}\right| \leq 1, \\ \pi \epsilon\left(0.8\left|x-x^{\prime}\right|^{-10}-2\left|x-x^{\prime}\right|^{-4}\right) & \text { otherwise, }\end{cases}
$$

which is derived by integrating along $y$ and $z$ the 12-6 LJ potential [29]. 


$$
s=1.4
$$
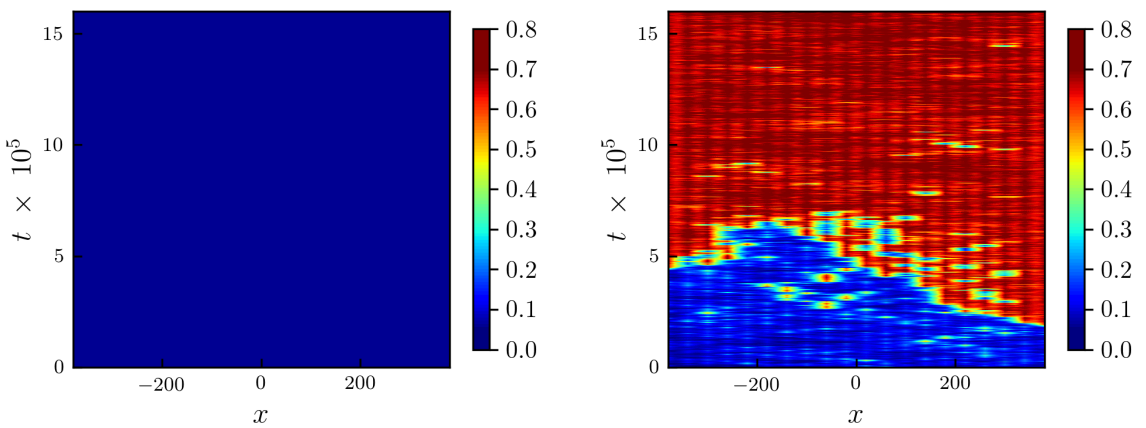

$$
s=1.8
$$
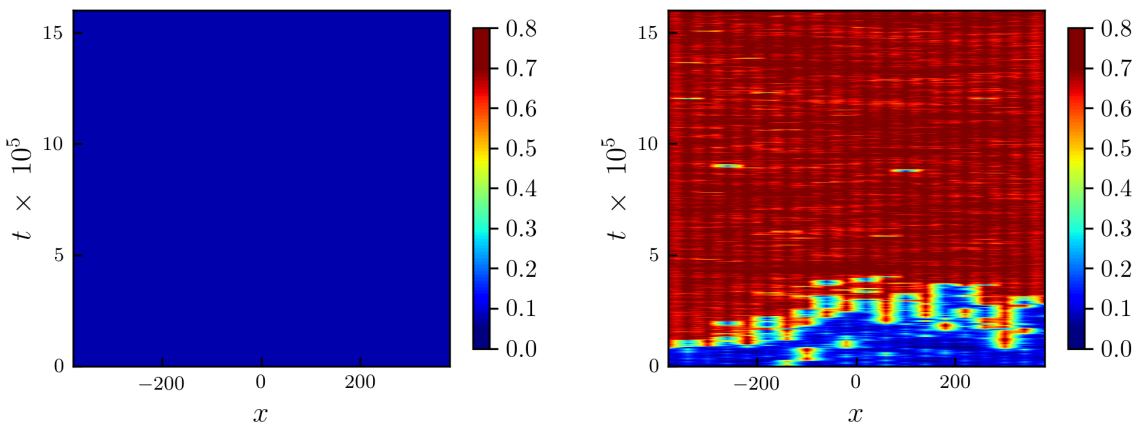

$$
s=2.2
$$
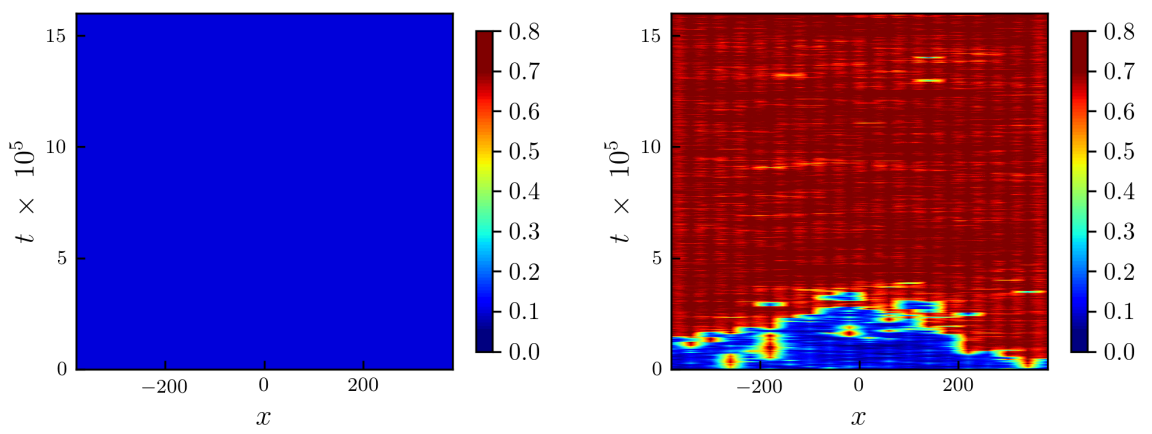

Fig. 10: Homogeneous nucleation of a vapour LJ system in metastable conditions with supersaturation ratio $s$. We report on the left column the mean field evolution, while on the right a single realization of the stochastic dynamics.

In order to analyse the vapour to liquid (first-order) phase transitions, we first compute the coexisting density profiles. The coexisting values of vapour and liquid density (binodal line) are denoted as $\rho_{v}$ and $\rho_{l}$ respectively, and are obtained by solving the following system of equations:

$$
\left\{\begin{array}{c}
\left.\frac{\partial \Omega}{\partial \rho}\right|_{\rho_{v}}=\left.\frac{\partial \Omega}{\partial \rho}\right|_{\rho_{l}}=0, \\
\Omega\left[\rho_{v}\right]-\Omega\left[\rho_{l}\right]=0 .
\end{array}\right.
$$

The meta-stable regions are delimited by the binodal and spinodal lines. The spinodal lines correspond to the inflection points of the grand free energy, hence are evaluated by solving:

$$
\left.\frac{\partial^{2} \Omega}{\partial \rho^{2}}\right|_{\rho_{v}}=\left.\frac{\partial^{2} \Omega}{\partial \rho^{2}}\right|_{\rho_{l}}=0 .
$$


(a)

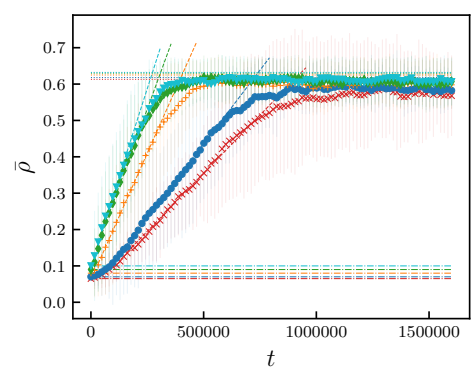

(b)

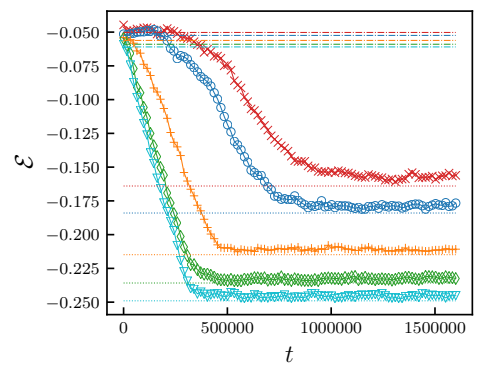

(c)

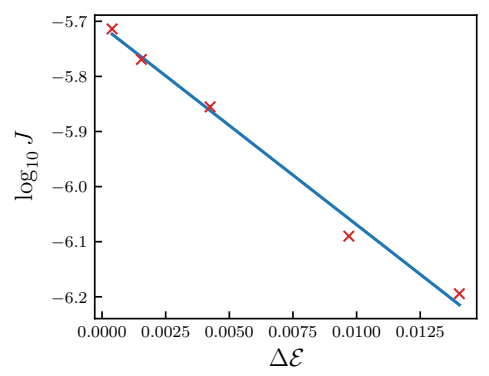

Fig. 11: Panel (a) reports the evolution in time of the average system density for the supersaturation ratios adopted in this study. Panel (b) shows the time-evolution of the system free energy for the supersaturation ratios adopted in this study. In (c), the nucleation growth rate is plotted against the free-energy barrier.

Finally, the bulk critical point is given by the intersection between binodal and spinodal lines, and thus is computed as

$$
\left.\frac{\partial \Omega}{\partial \rho}\right|_{\rho_{c}, T_{c}}=\left.\frac{\partial^{2} \Omega}{\partial \rho^{2}}\right|_{\rho_{c}, T_{c}}=0 .
$$

In Fig. 9(a), we report the bulk phase diagram obtained from the discretized grand free energy of the LJ fluid. Solid curves depicts the binodal, i.e. the locus of liquid-gas coexistence, while dashed curves depicts the spinodal, i.e. the boundary between the metastable and the unstable regions. The black circle designates the bulk critical point at $\rho_{c} \sim 0.3$ and $T_{c} \sim 1.35$.

If we denote with $\rho_{v}$ the vapour coexistence density at a given temperature, the supersaturation ratio is defined as $s=\rho / \rho_{v}$. We will study the nucleation of vapour systems with identical temperatures, but different initial supersaturation ratios. Figure 9(b) depicts the free-energy landscape as a function of the bulk density for such systems. At coexistence $s=1$, two stable basins are present, which means that the system has equal probability of being in one of the two. Increasing the supersaturation ratio, the high density wells, corresponding to the liquid phase, enhances its stability, thus leaving the vapour density in a metastable condition. Also, the energy barrier that the system has to overcome to pass from the vapour to the liquid phase decreases with $s$, until it becomes null at a supersaturation corresponding to the spinodal line. In such condition only one minimum of the grand free energy exists.

A physical description of the phase transition would consist of an initial and final uniform system densities, but also of a non-uniform density field during the transition. This means that the bulk grand-free energy in Fig. 9(b), being only valid for uniform densities, describes the system only in the initial and final stages, but it does not provide information on the transition path. The grand free energy for non-uniform systems is in general a function of each cell density, i.e. it is an $n$-dimensional manifold. To give a representative example of this, in Fig. 9 (c) we report our LJ grand-free energy for a non uniform system, constrained to have only two varying densities $\widetilde{\rho}_{1}=\left\{\rho_{1}=\cdots=\rho_{n / 2}\right\}$ and $\widetilde{\rho}_{2}=\left\{\rho_{n / 2+1}=\cdots=\rho_{n}\right\}$. The bulk free energy is then recovered for $\widetilde{\rho}_{1}=\widetilde{\rho}_{2}$ (dotted black line).

Single trajectories of the vapour to liquid phase transition, at different supersaturation ratios, are reported in Fig. 10. For comparison purposes, we perform simulations of the FDDFT and its mean field (deterministic) counterpart. In order for the transition to occur, the system grand free energy has to overcome an energy barrier. Such passage requires a local injection of energy, thus it is triggered by fluctuations. As a consequence, the mean field approach fails to describe the transition. Moreover, as expected by looking at the energy barrier in Fig. 9, the transition is favoured by higher supersaturation ratios.

In addition to the presence of fluctuations, the phase transition is allowed due to the open boundary conditions imposed in the system. These boundary conditions are described in subsection 3.5, and basically model the exchange of particles with a reservoir at constant temperature $T_{\text {res }}$ and chemical potential $\mu_{\text {res }}$. Thanks to them the mass of the system can increase (or decrease), thus permitting the transition from the lower-density minimum at the left of Fig. 9 (b) to the higher-density ones at the right. However, it is important to remark that these boundary conditions do not simply add (or remove) mass to the system. The imposed chemical potential at the boundary, $\mu_{\text {res }}$, can be iteratively 
solved to obtain the value of the density that satisfies it. We choose $\mu_{\text {res }}$ so that this iterative algorithm may converge to one of the two minima in Fig. 9 (b), depending on the initial conditions for the iteration. For the two simulations in Fig. 10 we always select to converge to the lower-density minimum in Fig. 9 (b). This is why, with identical boundary conditions, the mean field deterministic simulation at the left of Fig. 10 remains at the minimum of the left of Fig. 9 (b) and conserves the mass. On the contrary, the FDDFT simulation at the right of Fig. 10 is able to increase the mass thanks to the constant density at the boundary, which allows a continuous exchange of particles.

The trend observed in Fig. 10 is quantitatively analysed in Fig. 11, where we report the data obtained performing the ensemble average of 10 nucleation trajectories for each supersaturation ratio. Figure 11(a) shows the average density increase as a function of time. The initial and final average system densities are consistent with the vapour and liquid bulk densities predicted by the grand-free energy analysis.

The free energy evaluated at each time as function of the average density is reported in Fig. 11(b). The initial free-energy value, corresponding to the vapour metastable basin, evolves in time in order to the reach the more stable liquid basin, as predicted by Fig. 9(b). It is interesting to notice that the passage between the two basins implies a slight increase in the free energy due to the energy barrier overcome by the density field fluctuations.

We noticed that the average density kinetics is characterized by three main stages: 1) an initial latency period, 2) a growth period and, 3) an asymptotic relaxation towards a plateau, corresponding to the liquid phase density. This dynamics is consistent with the multi-stage nucleation pathway experimentally observed and theoretically studied in the phase-transition research community [74]. The growth period exhibits a linear-like trend, with slopes representing the nucleation growth rate $J$. As reported in the plot in Fig. 11(c), an Arrhenius like relation (as is the case with thermally activated processes) is observed between $J$ and the grand-free energy barrier $\Delta \mathcal{E}$, i.e.

$$
J \sim K \exp \left\{-\frac{\Delta \mathcal{E}}{T}\right\}
$$

where $J$ is the growth rate $K$ in the limit of a zero energy barrier. It is worth noticing that the pre-exponential factor $K$ in reality is not a constant, but can be often approximated as constant over limited supersaturation regions [75, 65].

Finally, we remark that the finite-volume scheme is able to accurately simulate processes where the number of particles per cell is greater than 5, as showed in subsection 4.1.1. For any process that involves smaller scales one has to rely on MD simulations. This could be relevant for certain processes such as nucleation, which may require capturing system features down almost to particle scales at initiation.

\section{Summary and conclusions}

We have developed an efficient and robust finite-volume numerical scheme for solving stochastic gradient flow equations, inspired by the ones from FDDFT, which also contributes to a better understanding of the effects of thermal fluctuations in physical phenomena. While previous works developed numerical methodology only applicable to a limited range of free energies (e.g. ideal-gas free energies such as in Refs [35, 71]), our proposed scheme deals effectively with general free-energy functionals, including for instance external fields or interacting potentials.

Our numerical methodology essentially comprises: a hybrid space discretization based on central and upwind schemes, for both deterministic and stochastic fluxes; a family of implicit-explicit Euler and Milsten time integrators, together with a weak second-order Runge-Kutta scheme; an adaptive time-step scheme, based on the Brownian bridge technique, which ensures the non-negativity of the density; and appropriate boundary conditions. The proposed scheme overcomes limitations of previous approaches in the literature. What is more, the hybrid approach provides an optimal compromise between statistical properties of the stochastic field and spurious oscillations. Additionally, our adaptive time-step scheme represents an alternative approach to preserve density positivity, without including artificial limiters.

In addition, we validate the proposed scheme by means of several numerical applications. First, we study the variance, temporal and spatial correlations, and structure factor of an ideal gas at equilibrium, comparing the results of our finite-volume solver with theoretical results from the literature and our own MD simulations. Consistently with previous works, we find that a minimum number of 5-10 particle per cell is required in order for FDDFT to match atomistic simulations results. We the examine the out-of-equilibrium evolution of an ideal gas in a doublewell external potential. As expected in this case, our stochastic solver accurately reproduces local mean density, local density fluctuations and spatial correlations obtained with MD simulations. We also highlight that, for the deterministic case/DDFT where thermal fluctuations are not included, the results are consistent with both FDDFT 
and MD. Finally, we simulate homogeneous nucleation kinetics of a fluid consisting of particles interacting through an LJ-like potential. Our results for the phase diagram match the theoretical results and serve so as to illustrate the crucial role of fluctuations to surmount free-energy barriers. Moreover, as expected, an exponential law is observed for the nucleation growth rate as function of the metastable free-energy barrier.

\section{Acknowledgments}

We gratefully acknowledge financial support from the Imperial College (IC) Department of Chemical Engineering PhD Scholarship scheme, IC President's PhD Scholarship scheme, ERC through Advanced Grant No. 247031 and EPSRC through Grants No. EP/L027186, EP/L020564 and EP/P031587/1. The computations were performed at the High Performance Computing center of IC. Finally, we are grateful to the anonymous reviewers for insightful comments and suggestions.

\section{References}

[1] A. Russo, M. Durán-Olivencia, S. Kalliadasis, R. Hartkamp, Macroscopic relations for microscopic properties at the interface between solid substrates and dense fluids, J. Chem. Phys. 150 (2019) 214705.

[2] L. Landau, E. Lifshitz, Statistical physics, vol. 5, Course of theoretical physics (1980).

[3] M. Bixon, R. Zwanzig, Boltzmann-langevin equation and hydrodynamic fluctuations, Phys. Rev. 187 (1969) $267-272$.

[4] R. F. Fox, G. E. Uhlenbeck, Contributions to non-equilibrium thermodynamics. i. theory of hydrodynamical fluctuations, Phys. Fluids 13 (1970) 1893-1902.

[5] K. T. Mashiyama, H. Mori, Origin of the landau-lifshitz hydrodynamic fluctuations in nonequilibrium systems and a new method for reducing the boltzmann equation, J. Stat. Phys. 18 (1978) 385-407.

[6] K. Kawasaki, Stochastic model of slow dynamics in supercooled liquids and dense colloidal suspensions, Physica A 208 (1994) 35 - 64.

[7] D. S. Dean, Langevin equation for the density of a system of interacting langevin processes, J. Phys. A: Math. Gen. 29 (1996) L613.

[8] P.-H. Chavanis, Hamiltonian and brownian systems with long-range interactions: V. stochastic kinetic equations and theory of fluctuations, Physica A 387 (2008) 5716-5740.

[9] P. Español, A. Donev, Coupling a nano-particle with isothermal fluctuating hydrodynamics: Coarse-graining from microscopic to mesoscopic dynamics, J. Chem. Phys. 143 (2015) 234104.

[10] A. J. Archer, M. Rauscher, Dynamical density functional theory for interacting brownian particles: stochastic or deterministic?, J. Phys. A 37 (2004) 9325-9333.

[11] M. A. Durán-Olivencia, P. Yatsyshin, B. D. Goddard, S. Kalliadasis, General framework for fluctuating dynamic density functional theory, New J. Phys 19 (2017) 123022.

[12] R. Evans, The nature of the liquid-vapour interface and other topics in the statistical mechanics of non-uniform, classical fluids, Adv. Phys. 28 (1979) 143-200.

[13] W. Dieterich, H. Frisch, A. Majhofer, Nonlinear diffusion and density functional theory, Z. Phys. B 78 (1990) $317-323$.

[14] B. D. Goddard, A. Nold, N. Savva, G. A. Pavliotis, S. Kalliadasis, General dynamical density functional theory for classical fluids, Phys. Rev. Lett. 109 (2012) 120603.

[15] B. Goddard, G. Pavliotis, S. Kalliadasis, The overdamped limit of dynamic density functional theory: Rigorous results, Multiscale Model. Sim. 10 (2012) 633-663.

[16] J. F. Lutsko, Recent developments in classical density functional theory, Adv. Chem. Phys. 144 (2010) 1.

[17] M. A. Durán-Olivencia, B. D. Goddard, S. Kalliadasis, Dynamical density functional theory for orientable colloids including inertia and hydrodynamic interactions, J. Stat. Phys. 164 (2016) 785-809.

[18] A. J. Archer, Dynamical density functional theory for molecular and colloidal fluids: A microscopic approach to fluid mechanics, J. Chem. Phys 130 (2009) 014509.

[19] U. M. B. Marconi, P. Tarazona, Dynamic density functional theory of fluids, J. Chem. Phys. 110 (1999) 8032-8044.

[20] B. D. Goddard, A. Nold, N. Savva, P. Yatsyshin, S. Kalliadasis, Unification of dynamic density functional theory for colloidal fluids to include inertia and hydrodynamic interactions: derivation and numerical experiments, J. Phys. Condens. Matter 25 (2012) 035101.

[21] A. Donev, E. Vanden-Eijnden, Dynamic density functional theory with hydrodynamic interactions and fluctuations, J. Chem. Phys. 140 (2014) 234115.

[22] J. F. Lutsko, A dynamical theory of nucleation for colloids and macromolecules, J. Chem. Phys. 136 (2012) 034509.

[23] J. F. Lutsko, M. A. Durán-Olivencia, Classical nucleation theory from a dynamical approach to nucleation, J. Chem. Phys. 138 (2013) 244908.

[24] M. A. Durán-Olivencia, P. Yatsyshin, S. Kalliadasis, J. F. Lutsko, General framework for nonclassical nucleation, New J. Phys. 20 (2018) 083019 .

[25] P. Yatsyshin, N. Savva, S. Kalliadasis, Density functional study of condensation in capped capillaries, J. Phys. Condens. Matter 27 (2015) 275104.

[26] B. D. Goddard, A. Nold, S. Kalliadasis, Dynamical density functional theory with hydrodynamic interactions in confined geometries, J. Chem. Phys. 145 (2016) 214106.

[27] A. Nold, B. D. Goddard, P. Yatsyshin, N. Savva, S. Kalliadasis, Pseudospectral methods for density functional theory in bounded and unbounded domains, J. Comp. Phys. 334 (2017) $639-664$.

[28] A. Nold, D. N. Sibley, B. D. Goddard, S. Kalliadasis, Fluid structure in the immediate vicinity of an equilibrium three-phase contact line and assessment of disjoining pressure models using density functional theory, Phys. Fluids 26 (2014) 072001. 
[29] P. Yatsyshin, N. Savva, S. Kalliadasis, Wetting of prototypical one- and two-dimensional systems: Thermodynamics and density functional theory, J. Chem. Phys. 142 (2015) 034708.

[30] P. Yatsyshin, A. O. Parry, C. Rascón, S. Kalliadasis, Wetting of a plane with a narrow solvophobic stripe, Mol. Phys. 116 (2018) $1990-1997$.

[31] J. F. Lutsko, How crystals form: A theory of nucleation pathways, Sci. Adv. 5 (2019) eaav7399.

[32] M. Kruger, D. S. Dean, A gaussian theory for fluctuations in simple liquids, J. Chem. Phys. 146 (2017) 134507.

[33] C. Villani, Topics in optimal transportation, 58, American Mathematical Soc., 2003.

[34] J. A. Carrillo, R. J. McCann, C. Villani, et al., Kinetic equilibration rates for granular media and related equations: entropy dissipation and mass transportation estimates, Rev. Mat. Iberoam. 19 (2003) 971-1018.

[35] C. Kim, A. Nonaka, J. B. Bell, A. L. Garcia, A. Donev, Stochastic simulation of reaction-diffusion systems: A fluctuating-hydrodynamics approach, J. Chem. Phys. 146 (2017) 124110.

[36] A. Donev, E. Vanden-Eijnden, A. Garcia, J. Bell, On the accuracy of finite-volume schemes for fluctuating hydrodynamics, Commun. Appl. Math. Comput. Sci. 5 (2010) 149-197.

[37] A. L. Garcia, M. M. Mansour, G. C. Lie, E. Cementi, Numerical integration of the fluctuating hydrodynamic equations, J. Stat. Phys. 47 (1987) 209-228.

[38] J. B. Bell, A. L. Garcia, S. A. Williams, Numerical methods for the stochastic landau-lifshitz navier-stokes equations, Phys. Rev. E 76 (2007) 016708 .

[39] J. B. Bell, A. L. Garcia, S. A. Williams, Computational fluctuating fluid dynamics, ESAIM: Math. Model. Numer. Anal. 44 (2010) 10851105.

[40] J. A. de la Torre, P. Español, A. Donev, Finite element discretization of non-linear diffusion equations with thermal fluctuations, J. Chem. Phys. 142 (2015) 094115.

[41] F. Balboa, J. B. Bell, R. Delgado-Buscalioni, A. Donev, T. G. Fai, B. E. Griffith, C. S. Peskin, Staggered schemes for fluctuating hydrodynamics, Multiscale Model. Sim. 10 (2012) 1369-1408.

[42] S. Delong, B. E. Griffith, E. Vanden-Eijnden, A. Donev, Temporal integrators for fluctuating hydrodynamics, Phys. Rev. E 87 (2013) 033302.

[43] V. Y. Glotov, V. M. Goloviznin, S. Karabasov, A. Markesteijn, New two-level leapfrog scheme for modeling the stochastic landau-lifshitz equations, Comput. Math. \& Math. Phys. 54 (2014) 315-334.

[44] R. Delgado-Buscalioni, G. De Fabritiis, Embedding molecular dynamics within fluctuating hydrodynamics in multiscale simulations of liquids, Phys. Rev. E 76 (2007) 036709.

[45] G. De Fabritiis, M. Serrano, R. Delgado-Buscalioni, P. Coveney, Fluctuating hydrodynamic modeling of fluids at the nanoscale, Phys. Rev. E 75 (2007) 026307.

[46] G. De Fabritiis, R. Delgado-Buscalioni, P. Coveney, Multiscale modeling of liquids with molecular specificity, Phys. Rev. Lett. 97 (2006) 134501 .

[47] S. A. Williams, J. B. Bell, A. L. Garcia, Algorithm refinement for fluctuating hydrodynamics, Multiscale Model. Sim. 6 (2008) 1256-1280.

[48] A. Donev, J. B. Bell, A. L. Garcia, B. J. Alder, A hybrid particle-continuum method for hydrodynamics of complex fluids, Multiscale Model. Sim. 8 (2010) 871-911.

[49] A. K. Bhattacharjee, K. Balakrishnan, A. L. Garcia, J. B. Bell, A. Donev, Fluctuating hydrodynamics of multi-species reactive mixtures, J. Chem. Phys. 142 (2015) 224107.

[50] P. J. Atzberger, Spatially adaptive stochastic numerical methods for intrinsic fluctuations in reaction-diffusion systems, J. Comput. Phys. 229 (2010) 3474-3501.

[51] M. A. Durán-Olivencia, R. S. Gvalani, S. Kalliadasis, G. A. Pavliotis, Instability, rupture and fluctuations in thin liquid films: Theory and computations, J. Stat. Phys. 174 (2019) 579-604.

[52] F. Otto, Double degenerate diffusion equations as steepest descent, Sonderforschungsbereich 256, 1996.

[53] C. Villani, Topics in optimal transportation, 58, American Mathematical Soc., 2003.

[54] C. Reina, J. Zimmer, Entropy production and the hgeometry of dissipative evolution equations, Phys. Rev. E 92 (2015) 052117.

[55] A. B. Barbaro, J. A. Canizo, J. A. Carrillo, P. Degond, Phase transitions in a kinetic flocking model of cucker-smale type, Multiscale Model. Sim. 14 (2016) 1063-1088.

[56] J. A. Carrillo, R. J. McCann, C. Villani, Contractions in the 2-wasserstein length space and thermalization of granular media, Arch. Ration. Mech. Anal. 179 (2006) 217-263.

[57] J. A. Carrillo, K. Craig, F. S. Patacchini, A blob method for diffusion, Calc. Var. Partial Diff. 58 (2019) 53.

[58] M. Paulus, C. Gutt, M. Tolan, Static structure factor of capillary waves at large momentum transfer, Phys. Rev. B 78 (2008) 235419.

[59] A. O. Parry, C. Rascón, R. Evans, The local structure factor near an interface; beyond extended capillary-wave models, J. Phys. Condens. Matter 28 (2016) 244013.

[60] K. Mohamed, M. Seaid, M. Zahri, A finite volume method for scalar conservation laws with stochastic time-space dependent flux functions, J. Comput. Appl. Math. 237 (2013) 614-632.

[61] P. K. Sweby, High resolution schemes using flux limiters for hyperbolic conservation laws, SIAM J. Numer. Anal. 21 (1984) $995-1011$.

[62] J. A. Carrillo, A. Chertock, Y. Huang, A finite-volume method for nonlinear nonlocal equations with a gradient flow structure, Comm. Comput. Phys. 17 (2015) 233-258.

[63] K. Balakrishnan, A. L. Garcia, A. Donev, J. B. Bell, Fluctuating hydrodynamics of multispecies nonreactive mixtures, Phys. Rev. E 89 (2014) 013017

[64] P. E. Kloeden, E. Platen, Numerical Solution of Stochastic Differential Equations, Springer-Verlag Berlin Heidelberg, 1992.

[65] J. F. Lutsko, M. A. Durán-Olivencia, A two-parameter extension of classical nucleation theory, J. Phys. Condens. Matter 27 (2015) 235101.

[66] B. Øksendal, Stochastic differential equations, in: Stochastic differential equations, Springer, 2003, pp. 65-84.

[67] M. Bessemoulin-Chatard, F. Filbet, A finite volume scheme for nonlinear degenerate parabolic equations, SIAM J. Sci. Comput. 34 (2012) B559-B583.

[68] J. G. Gaines, T. J. Lyons, Variable step size control in the numerical solution of stochastic differential equations, SIAM J. Appl. Math. 57 (1997) 1455-1484.

[69] V. Sotiropoulos, Y. N. Kaznessis, An adaptive time step scheme for a system of stochastic differential equations with multiple multiplicative noise: chemical langevin equation, a proof of concept, J. Chem. Phys. 128 (2008) 014103. 
[70] S. Plimpton, Fast parallel algorithms for short-range molecular dynamics, J. Comput. Phys. 117 (1995) 1-19.

[71] N. K. Voulgarakis, J.-W. Chu, Bridging fluctuating hydrodynamics and molecular dynamics simulations of fluids, J. Chem. Phys. 130 (2009) $04 \mathrm{~B} 605$.

[72] J. A. Barker, D. Henderson, Perturbation theory and equation of state for fluids. ii. a successful theory of liquids, J. Chem. Phys. 47 (1967) 4714-4721.

[73] N. F. Carnahan, K. E. Starling, Equation of state for nonattracting rigid spheres, J. Chem. Phys. 51 (1969) $635-636$.

[74] M. A. Durán-Olivencia, P. Yatsyshin, S. Kalliadasis, J. F. Lutsko, General framework for nonclassical nucleation, New J. Phys. 20 (2018) 083019.

[75] J. F. Lutsko, M. A. Durán-Olivencia, Classical nucleation theory from a dynamical approach to nucleation, J. Chem. Phys. 138 (2013) 244908.

\section{Appendix A. MD simulations details}

MD simulations are performed using the Large-Scale Atomic/Molecular Massively Parallel Simulator (LAMMPS) [70]. Particle positions and velocities are integrated in time using the velocity-Verlet algorithm, with a time-step of $d t=0.001 \tau$. The system is simulated at constant temperature and volume, so that particle coordinates are consistent with the canonical ensemble (NVT). Specifically, the temperature $T=1$ is kept constant during the simulations using a Langevin thermostat. All the physical quantities are expressed in reduced units, i.e. they are nondimensionalized with the fundamental quantities $\sigma, \epsilon$ and $m$, representing distance, energy and mass, respectively. Further, without loss of generality, $\sigma, \epsilon, m$ and the Boltzmann constant $k_{B}$ are set equal to unity.

As discussed extensively in [1], a macroscopic field $X(\mathbf{r}, t)$ can be extracted from particle coordinates as $X(\mathbf{r}, t)=$ $\sum_{i} \chi_{i} \phi\left(\mathbf{r}_{i}(t)-\mathbf{r}\right)$, where $\chi_{i}$ is the information of interest of particle $i$ at position $\mathbf{r}_{i}$ at time $t$, and $\phi$ is a kernel function(commonly a piecewise constant, Gaussian, or polynomial function). In this work, we adopt a piecewise constant function defined as:

$$
\phi(y)= \begin{cases}\frac{1}{\Delta x} & \text { for }\|y\|<\Delta x / 2, \\ 0 & \text { otherwise }\end{cases}
$$

with $\Delta x$ being the width of each bin. In each comparison, we match the $\Delta x$ for MD simulations with the one for the discretized FDDFT. Using the above, the instantaneous macroscopic density profile for a single trajectory is computed as:

$$
\rho(x, t)=\sum_{i} m_{i} \phi\left(x_{i}(t)-x\right)
$$

where $m_{i}$ is the mass of the particle $i$.

Equilibrium simulations. MD simulations of ideal gas fluids in equilibrium are performed using a fixed number of particles $(1,000)$ in a 1D domain of length 2,000 (in reduced units) with periodic boundary conditions. The system is equilibrated and then a run of $2 \times 10^{7}$ time steps is performed, during which fluid particle positions and velocities are stored every $10^{4}$ time steps for analysis. The process is repeated $10^{3}$ times to generate independent trajectories.

Non-equilibrium simulations. MD simulations of ideal gas fluids in non-equilibrium conditions are performed using a fixed number of particles (200) in a one-dimensional (1D) domain of length 200 (in reduced units) with periodic boundary conditions, under an external potential:

$$
V(x)=5\left[\left(\frac{x}{200}\right)^{4}-\left(\frac{x}{200}\right)^{2}\right] .
$$

A run of $2 \times 10^{6}$ time steps is performed, during which fluid particle positions and velocities are stored every $10^{3}$ time steps for analysis. The process is repeated $10^{3}$ times with different (random) initial conditions to generate independent trajectories and gather statistics. 


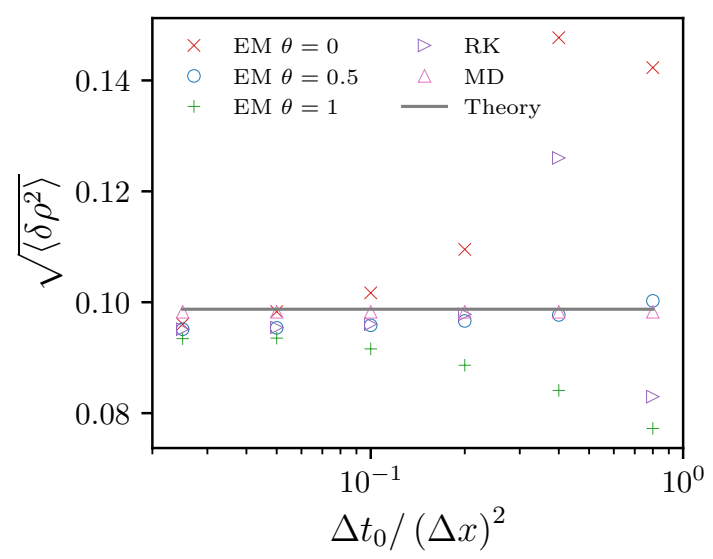

Fig. B.12: Standard deviation $\sqrt{\left\langle\delta \rho^{2}\right\rangle}$ as a function of the initial time-step $\Delta t_{0}$ for an ideal gas in equilibrium. Temporal integrators. EM: Euler-Maruyama, MI: Milstein, RK: Runge-Kutta, MD: Molecular dynamics. Explicit $(\theta=0)$, semi-implicit $(\theta=0.5)$ and implicit $(\theta=1)$, MD: Molecular dynamics, Theory: Eq. (71).

\section{Appendix B. Time integrators stability analysis}

Both stability and accuracy of the different time-integrators are relevant, given that large time-steps are required in many applications (for instance, for transitions occurring over long time-scales). In the main text, we focused on the accuracy of the schemes comparing finite-volume schemes, MD and theoretical results. Here we analyze the stability of the different time integrators with respect to the time-step size.

Specifically, in Fig. B.12 we report a comparison of the fluctuations standard deviation obtained with some selected time integrators and the MD-theoretical results for varying time step sizes $\Delta t_{0}$. It is worth underlying that, because of the adaptive time step adopted in the simulations, $\Delta t_{0}$ represents only the initial time-step and the effective time-step may not be constant throughout the simulations, i.e. it may be lower than $\Delta t_{0}$. The system considered here is the same ideal gas system (with average density $\bar{\rho}=0.5$ ) used for the analyses in the main text. The cell size adopted is $\Delta x=50$, corresponding to a number of particles per cell $N_{c}=25$. Moreover, we do not report the results for the Milstein schemes, since in previous tests we did not observe any relevant difference between the Milstein scheme and the Euler-Maruyama one as far as the mean, variance and correlations are concerned. Figure B.12 shows that the semi-implicit scheme outperforms both explicit and implicit schemes at high $\Delta t_{0} /(\Delta x)^{2}$, becoming the time-integrator of choice for computations requiring large time-steps. Moreover, the explicit Runge-Kutta scheme shows an enhanced stability compared to both implicit and explicit Euler-Maruyama. 